\title{
Ultrafine Cohesive Powders: From Interparticle Contacts to Continuum Behaviour
}

\author{
Rostyslav Tykhoniuk $^{*(1)}$, Jürgen Tomas ${ }^{(1)}$, Stefan Luding ${ }^{(2)}$, Michael Kappl ${ }^{(3)}$, Lars Heim ${ }^{(3)}$, \\ Hans-Jürgen Butt ${ }^{(3)}$
}

(1) Mechanical Process Engineering, Otto-von-Guericke University of Magdeburg, Universitätsplatz 2, D - 39106 Magdeburg, Germany

(2) Particle Technology, Nanostructured Materials, DelftChemTech, TU Delft, Julianalaan 136, 2628 BL Delft, Netherlands

(3) Max-Planck-Institute for Polymer Research, Ackermannweg 10, 55128, Mainz, Germany

\begin{abstract}
Continuum mechanical models and appropriate measuring methods to determine the materials parameters are available to describe the flow behaviour of cohesive powders. These methods are successfully applied to design process equipment as silos. In addition, "microscopic" studies on the particle mechanics can give a better physical understanding of essential "macroscopic" constitutive functions describing a powder "continuum". At present, by means of the discrete element method (DEM), a tool is available that allows one to consider repulsive and frictional as well as attractive adhesion forces in detail. Within the framework of Newton's equations of motion, each particle in the system is tracked, and reacts to the forces acting.

The knowledge of the interaction forces between particles is thus a prerequisite for understanding (via DEM) the stability and flow of particulate systems and other phenomena. In this study, macroscopic cohesion and friction are related to their microscopic counterparts, adhesion and contact-friction. The macroscopic cohesion is found to be proportional to the maximal microscopic adhesion force, and the macroscopic friction coefficient is a non-linear function of the contact friction, dependent (or independent) on the preparation procedure for yielding (or steady-state flow).

One of the few methods available for the direct measurement of surface- and contact-forces is the atomic force microscope (AFM) and, related to it, the so-called particle interaction apparatus (PIA). A contact model for ultrafine cohesive particles (average radius $\mathrm{d}_{50} \approx 1 \mu \mathrm{m}$ ) is introduced, based on such experiments. Plugged into DEM, consolidation, incipient yielding, and steady-state flow of the model powders are studied. Also the dynamic formation of the shear zone is examined and compared with experimental observations. Eventually, the shear experiments with volumetric strain measurements in a translational shear cell are used for validation.
\end{abstract}

\footnotetext{
* Corresponding author. Tel.: +49-391-67-12590; fax: +49-391-67-11160.

E-mail: Rostyslav.Tykhoniuk@vst.uni-magdeburg.de
} 


\section{INTRODUCTION}

Known flow problems of cohesive particulate solids (flow function according to Jenike (1964) $\left.f f_{c}<4\right)$ in storage and transportation containers, conveyors or process equipment, include bridging, channelling, segregation, flooding, and avalanching. In addition, the insufficient apparatus- and system-reliability of solid processing plants is also related to such flow problems. This (incomplete) list of technical problems and hazards motivates us to deal with the fundamentals of cohesive powder consolidation and flow behaviour, i.e., to develop a reasonable combination of particle and continuum mechanics. The goal of the present work is to build a bridge between the "microscopic" particle interaction properties, similar to Castellanos (2005), but here based on AFM/PIA measurements, and the macroscopic constitutive behaviour of cohesive powder flows, involving models of elastic-plastic-adhesive contact behaviour.

Continuum mechanical models and appropriate measuring methods are successfully applied to describe the flow behaviour of cohesive powders, as well as for the practical design of process equipment like silos. However, the essential constitutive functions of the powder "continuum" can be more deeply described and understood with the help of particle mechanics (Tomas, 2001). The Discrete Element Method (DEM) (Cundall, 1979) is an alternative solution tool, which allows us to take into account the contact properties and, what is especially important in our case, adhesion forces. We will introduce these into the equations of motion of the particles and study the dynamic behaviour of cohesive powders with the goal of a "microscopic" understanding.

The interaction force between two macroscopic bodies can be measured by the spring balance method as used by Bradley (1932), and then by Derjaguin et al. (1956), Black et al. (1960), Rouweller et al. (1971), Israelachvili and Tabor (1972, 1973), Israelachvili and Adams (1978) and Israelachvili (1992). The spring balance method is not helpful for too small particles. With a micron-sized particle glued to the end of an AFM cantilever as force sensor, one can measure the adhesion and friction forces at the contact. The atomic force microscope (AFM), invented by Binnig, Quate and Gerber (1986), has become an important tool for imaging the topography of surfaces with high resolution. Soon after the invention of the AFM it was realised that it can also be used for force-versus-distance measurements (Meyer et al, 1989; Weisenhorn et al, 1989). Data from such force-distance curves are useful to study all kinds of 
surface forces or material properties like elasticity. For reviews on AFM force measurements see Butt, Jaschke and Ducker (1995) and Capella and Dietler (1999).

Combining the knowledge about macroscopic shear tests of cohesive powders with the microscopic AFM measurements of the particle interaction forces, it is possible to develop a contact constitutive model that describes the deformation behaviour of ultrafine, cohesive, frictional particles. While much attention was paid to tangential and rolling or torsion degrees of freedom recently, see Bartels et al. (2005) and Luding (2007), in this study we mainly focus on the adhesive and plastic normal contact forces. In this context, an irreversible inelastic contact flattening is of vital importance, as an essential element and physical reason of a load-dependent adhesion due to van der Waals forces. A mean-field microscopic model for contacts with elastic, plastic, and adhesion forces was proposed, as based on macroscopic observations from bulk experiments (Tomas, 2001; Tomas, 2002). The contact-model in a simplified form is applied to model powders in the framework of DEM simulations - to two different systems: the Jenike shear test and the biaxial shear box - in order to find out the relationship between the mechanical parameters of a single particle contact on a microscopic level, and the macroscopic flow parameters of the powder constitutive relations on the level of a continuum theory.

The structure of the paper follows the micro-macro philosophy outlined above. In section 2, the contact force experiments are reviewed and discussed, while section 3 contains the contact models used later in the particle simulation approach, as described in section 4. Section 5 describes the model systems examined and section 6 deals with the reference experiments. Simulation results are the focus of section 7 and the key findings of this paper are summarized and discussed in section 8 . 


\section{DIRECT MEASUREMENT OF CONTACT (ADHESION) FORCES}

In the following, contact-force measurement techniques are reviewed in subsections 2.1 and 2.2, where special attention is paid to force displacement measurements (2.3) and sample preparation (2.4). Adhesion force results are presented in subsection 2.5 and some results on friction forces are presented in 2.6.

\subsection{Centrifugal Testing Techniques}

Adhesion forces between particles and planar surfaces have been measured with the centrifugal method since more than 40 years (Larsen 1958; Kordecki 1960; Böhme 1962; Polke 1968; Polke 1969). A significant part of the knowledge about the contact behaviour of powders stems from such experiments. Usually the detachment force of many particles is measured in a single experiment, allowing statistical evaluation of the data. This is especially useful in the case of irregular shaped particles where the contact area and adhesion force will depend on the random orientation of the particles relative to each other or to the surface. Therefore, the centrifuge technique is a standard method to characterise the behaviour of industrial powders in pharmaceutical (Newton 1992a; Newton 1992b; Newton 1993; Podczeck 1994; Podczeck 1995a; Podczeck 1995b; Podczeck 1997a; Podczeck 1997b; Podczeck 1999) or food applications (Rennie 1998). By tilting the surfaces to which the particles are attached, the centrifuge technique can also be used to study friction forces (Podczeck 1995c; Podczeck 1995d). There are, however, also disadvantages of this technique. One limit is that the rotational speed of the available ultra-centrifuges is limited due to the material stability of the rotor. This restricts adhesion measurements with the centrifuge method to particles larger than a few $\mu \mathrm{m}$. Otherwise, the centrifugal force is not strong enough to detach the adhering particles from the surface. Additionally, the results depend directly on the particle size because of the volume force used to detach the particle from surface. This can be avoided when one measures the particle-particle or particle-surface interaction forces with the so-called colloidal probe technique.

\subsection{Principle of direct force displacement measurement}

The colloidal probe technique, or, in other words, direct assessment of the particle-particle or particle-surface interaction forces, is possible with the atomic force microscope. Using a 
micron-sized particle glued to the end of an AFM cantilever as the force sensor, this technique was developed for the study of colloidal interactions (Kappl 2002). When comparing measured force-distance profiles (between an AFM tip and a surface) to theoretical models, one encounters the problem of the poorly defined geometry of the AFM tip. An approach to get a better defined geometry has been proposed by Hüttl (1997). They etched silicon AFM tips in an oven in the presence of oxygen and obtained tips with a spherical end of well defined radius. A more universal general solution is to replace the tip by a colloidal particle with well defined spherical shape and known radius, as shown in Fig. 1.

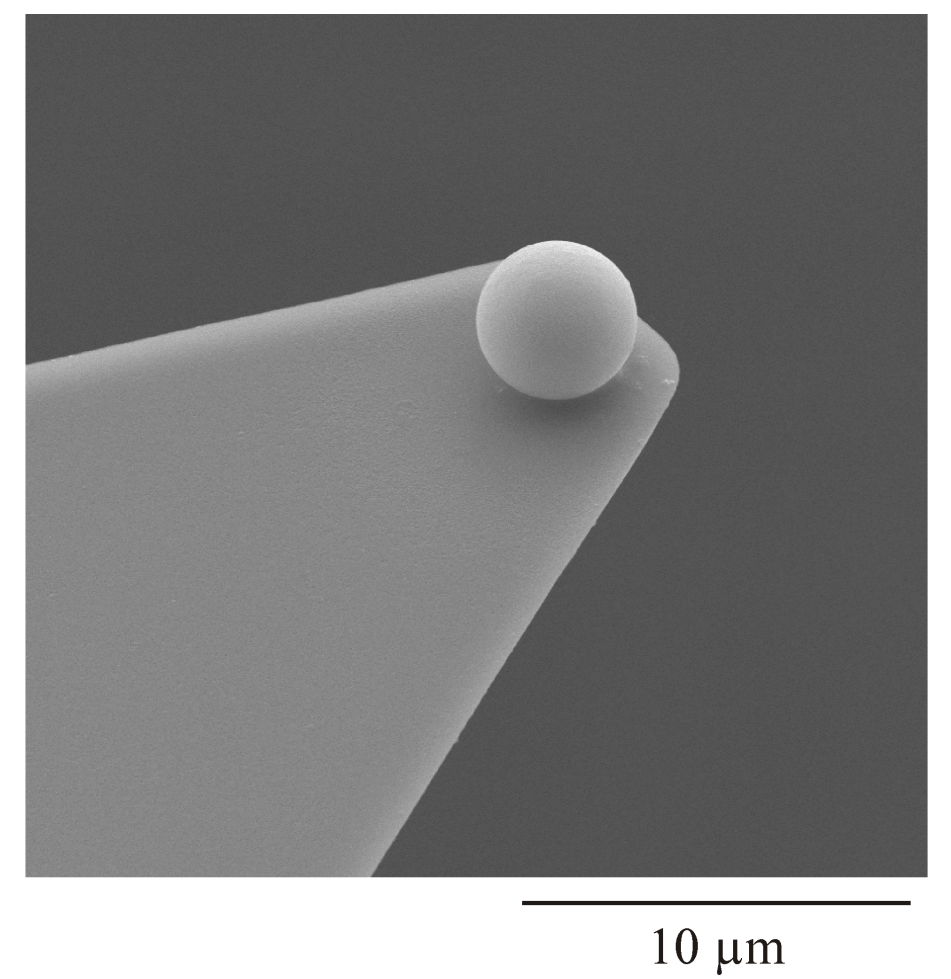

Fig. 1: Scanning electron micrograph of a silanized silica microsphere glued to the end of a tipless atomic force microscope cantilever (Kappl 2002)

This so-called "colloidal probe technique" was first applied by Ducker $(1991,1992)$ and Butt (1991) and since then it has become a well-established and powerful tool for the study of surface forces. Its measuring principle predestines it for the investigation of particle interactions, making single particle contact experiments feasible.

The colloidal probe technique offers the advantage that the same particle can be used for a series of experiments and its surface can be examined afterwards. However, there are also 
disadvantages. Typically, the accessible particle size is limited to the range between $1 \mu \mathrm{m}$ and $50 \mu \mathrm{m}$, and the tedious sample preparation limits the number of different particles that can be used within one study. Therefore the two methods (centrifuge and "colloidal") complement each other in the research of particle systems. Up to our knowledge, there has not been any study, up to now, that directly compared the centrifuge technique and the colloidal probe technique. However, both methods have provided valuable results that are in agreement with the established theories of contact mechanics. Here we describe the principles of the colloidal probe technique concerning one special application: the measurement of adhesion forces. In order to understand the flow behaviour of a powder not only the adhesion but also the sliding and rolling friction needs to be known. First experiments have been done to measure also these tangential forces with the AFM (Heim 1999; Cain 2000; Feiler 2000; Ecke 2001a; Ecke 2001b; Zauscher 2001) although they are less systematic than the adhesion experiments.

\subsection{Direct force displacement measurement in detail}

The measuring principle of the colloidal probe technique is identical to that of a standard AFM as outlined in the caption of Fig. 2. The sample is moved by applying a voltage to the piezoelectric translator onto which the sample is mounted. The deflection of the cantilever is normally measured using the optical lever technique: A beam from a laser diode is focused onto the end of the cantilever and a position sensitive detector monitors the position of the reflected beam. The backside of the cantilever is usually covered with a thin gold layer to enhance its reflectivity. When a force is applied to the probe, the cantilever bends and the reflected light-beam moves on the detector. 


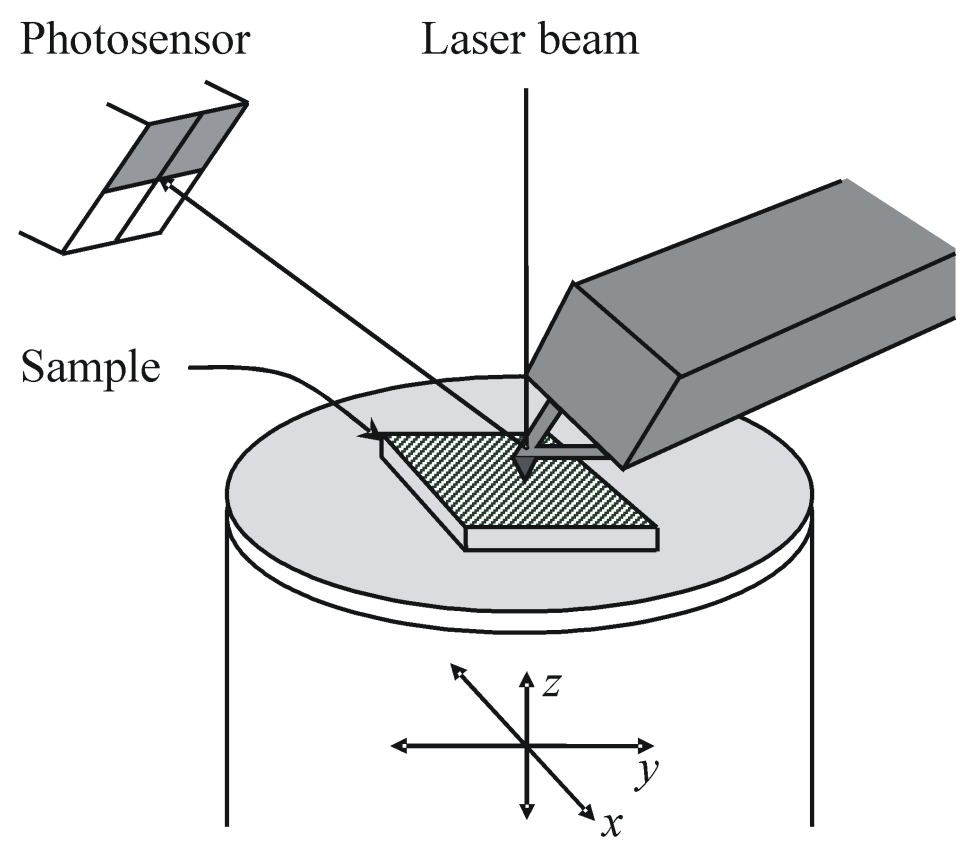

Piezoelectric scanner

Fig. 2: Principle of an AFM. The sample is mounted onto a piezoelectric scanner and can be raster-scanned while (almost) in contact with the sharp tip that sits at the end of the cantilever. The deflection of the cantilever is detected with an optical lever technique. A laser beam is reflected from the backside of the cantilever onto a split photodiode, and the change in position of the laser spot is recorded.

The direct result of such a force measurement is the detector signal in volts, $\Delta V$, as function of the position of the piezo, $\Delta z_{p}$, normal to the surface (Fig. 3a). To obtain a force- displacement curve, $\Delta V$ and $\Delta z_{p}$ have to be converted. To calculate the cantilever deflection from the detector signal, the corresponding conversion factor is needed, which can be obtained from a linear fit of the "constant compliance" region. The tip-sample separation is then obtained by adding the cantilever deflection to the piezo position. The force, acting on the cantilever, $F$, is obtained by multiplying its deflection with the spring constant of the cantilever (Fig. 3b). 

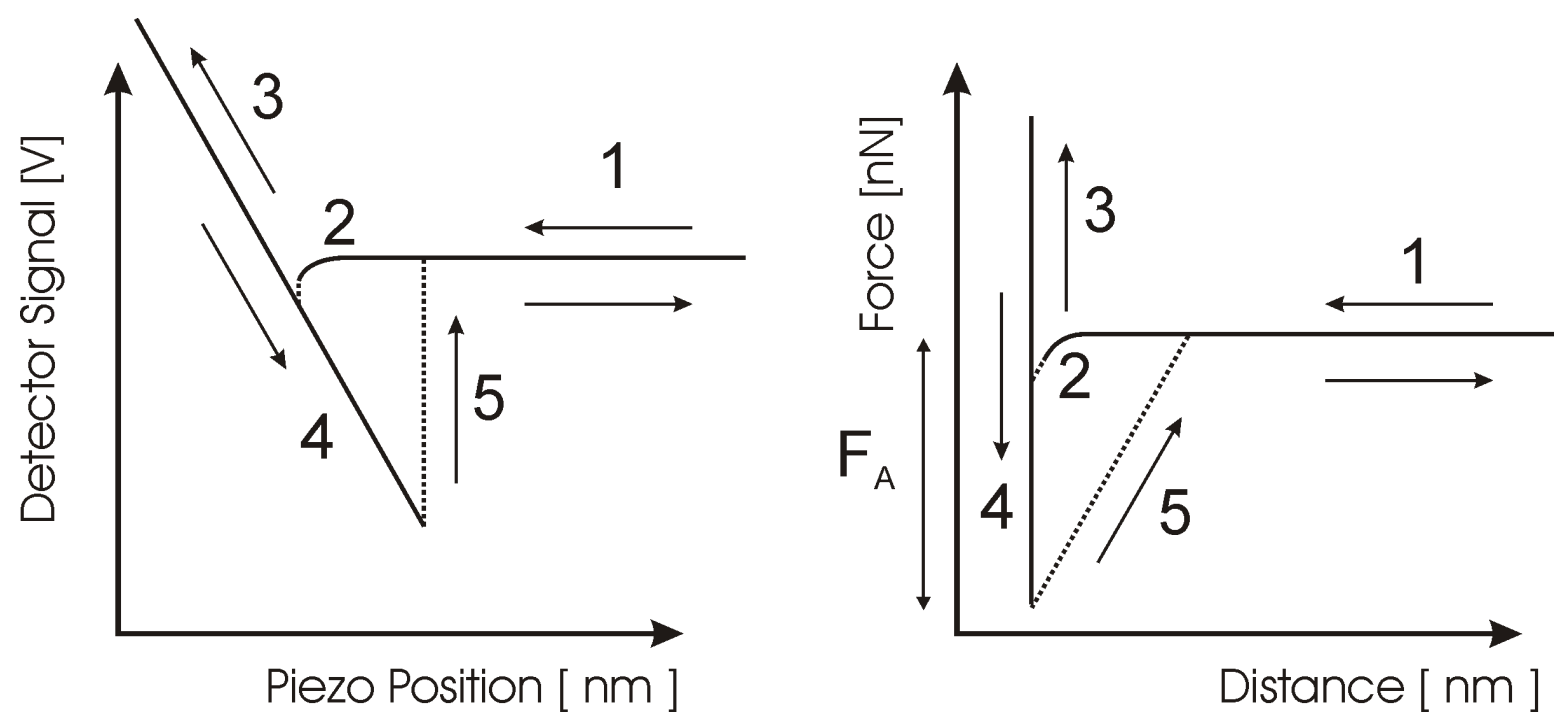

Fig. 3: a) Schematic of a deflection signal plotted against the piezo position. When the colloidal probe is approaching, but still far from the surface, no deflection will occur (1). When the probe gets close to the substrate, the (in this case attractive) surface forces will cause a bending of the cantilever towards the surface (2). As soon as the attractive force gradient becomes larger than the spring constant of the cantilever, the probe jumps in contact with the surface. From this moment, probe and surface will move in parallel (assuming that the deformations of the surfaces and at the contacts are negligible). The resulting straight line corresponds to the so called "constant compliance" region (3). On retreat, the probe will usually adhere to the surface, causing the cantilever to bend downwards (4). Eventually the bending force will become larger than the adhesion or pull-off force, and the cantilever will snap off the surface into its equilibrium position (5). b) Corresponding force-distance curve after multiplying the deflection with the calibration coefficient obtained from a linear fit of the cantilever, and adding the cantilever deflection to the piezo position. $F_{A}$ denotes the (pulloff) adhesion force.

For the colloidal probe technique, standard AFMs can be employed, and most results were obtained with commercial instruments. Some groups have chosen to build custom made devices optimised for this technique (Butt 1994; Pierce 1994; Craig 1996; Preuss 1998; Braithwaite 1997; Toikka 2001), because standard AFMs have some technical limitations that make them inadequate for colloidal probe experiments. The tip apex of an AFM tip typically has a radius of $5-50 \mathrm{~nm}$, whereas the radii of colloidal probes are in the range of $1-50 \mu \mathrm{m}$, resulting in much higher adhesion forces. Due to the limited range of piezo-movement and the 
dynamic range of the split photodiode detector, considerably stiffer cantilevers have to be used to ensure detachment of the particle after contact. This results in a loss of force and distance resolution. Other problems are creep and hysteresis of the piezos, which may cause distortion of the force curves, especially at the point of reversal of the piezo movement. Possible improvements involve the replacement of the split photodiode by a position sensitive device, which has a much higher dynamic range (Butt 1994; Pierce 1994; Preuss 1998), and the use of piezos with a higher scanning range and closed loop position control (Preuss 1998).

\subsection{Sample preparation - attachment of particles}

Before a colloidal probe experiment is performed, the particle probe has to be attached to the end of the cantilever. In principle, any kind of material that can be glued to the cantilever can be used. For practical reasons, often materials are preferred that are available in the form of spherical particles with smooth surfaces. This facilitates comparison with theoretical models und minimizes the influence of surface roughness, but also limits the number of usable materials. Colloidal particles are usually attached by micromanipulators while under optical control with an optical microscope, thus limiting the minimal particle size to $\sim 1 \mu \mathrm{m}$. An attempt to introduce a "nano-colloidal probe" technique has been presented recently by (Cho 2002). Basically, a tiny amount of glue $\left(\sim 10^{-18} \mathrm{~m}^{3}\right)$ is placed onto the very end of the cantilever and then the colloidal probe is brought in contact with this spot. This is accomplished either by keeping the cantilever fixed and using fine wires to move the glue and particles (Ducker 1992), or to pick up the glue and the particle by moving the cantilever (Raiteri 1998). Most common types of glue are epoxy resins like Epicote 1004 or Epon $\mathrm{R}$ 1004F resin (Shell) (Ducker 1992), UV-curable glues like Norland Optical Cement No. 68 (Schaefer 1995) or Loctite glass bond (Bowen 2000). For some materials, e.g., polystyrene (Schaefer 1994), or borosilicate glass (Bonaccurso 2005), sintering of the particles to the cantilever is feasible, avoiding possible surface contamination by the glue.

\subsection{Adhesion force measurement setup}

The adhesion between surfaces is governed by the deformation of the two bodies in contact, and the surface forces acting between them. These two phenomena are inherently coupled as the deformation will depend on the acting forces and at the same time the surface forces will 
depend on the geometry of the bodies. This interdependence makes the theory of adhesion a complex problem that is still under debate.

Most experimental results on adhesion forces were analysed based on the JKR model by Johnson, Kendall and Roberts (1971) (JKR model) or the DMT model by Derjaguin, Muller and Toporov (1975). The JKR model predicts the adhesion between particles and a substrate, assuming that all materials deform elastically and interactions occur within the contact zone only. In the DMT model, surface interactions outside the contact zone are included, predicting somewhat larger effective contact radii and adhesion forces. For both JKR and DMT model the adhesion forces depend linearly on the (reduced) particle radius, Eq. (1). Therefore, adhesion force values are often given as a reduced force, i.e. the contact force divided by the (reduced) radius of the particles. The adhesion force is

$$
F_{a d h} \propto R \quad \text { with } \quad R=\frac{R_{1} R_{2}}{R_{1}+R_{2}}
$$

where $R_{1}$ and $R_{2}$ are the radii of the two particles in contact. For small, micron-sized particles, these idealized continuum theories may fail as the contact deformations get down to a molecular scale. We investigated the dependence of adhesion between single silica spheres with reduced radii between $0.35 \mu \mathrm{m}$ and $1.3 \mu \mathrm{m}$ (Heim 1999). Fig. 4 shows that even for such small values of $R$ a linear dependence between $F_{a d h}$ and $R$ is observed, as predicted by the JKR and DMT theories. 


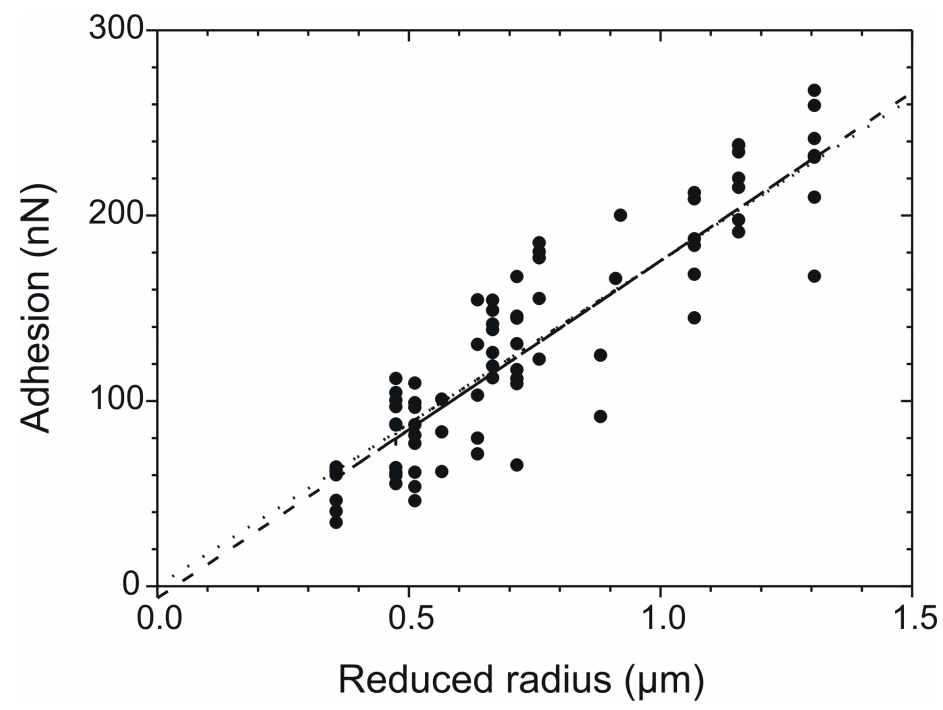

Fig. 4: Dependence of the adhesion force between pairs of silica particles on the reduced radius. Each data point is an average of seven adhesion measurements obtained with one pair of microspheres. The dashed line is a linear regression fit of the measured data, and the dotted line is the best linear fit of the data that passes through the origin with slope $F_{a d h} / R=0.174$ $\mathrm{N} / \mathrm{m}$.

No dependence of the adhesion force on the applied load or the contact time is expected in the DMT and JKR models. In practice this will hold only as long as the deformations remain purely elastic, i.e., reversible. If one of the materials shows non-elastic (plastic) deformation, the contact area and thus the adhesion force will increase with the applied normal force or, possibly, with the contact time. Therefore, the dependence of adhesion on the applied load and contact time can reveal much about the contact mechanics details and the mechanical properties of the materials involved.

A common observation in adhesion force measurements using the colloid probe technique is the enormous scatter of the data. Each data point in Fig. 4 corresponds to an average of at least seven force measurements between one pair of particles. The error bars for this average are about as large as the single data points. When using a different pair of particles with the same reduced radius, a strongly different adhesion force is typically observed. This is not a limitation of the experimental method, but reflects the actual difference in adhesion force due to surface inhomogeneities such as surface roughness or local variation in surface chemistry. When switching from the almost "ideal" model system of silica spheres to more application relevant, technical powders, an even wider distribution of adhesion values is observed. An 
example is shown in Fig. 5, where adhesion forces between carbonyl iron powder (CIP) particles are shown (Heim 2005). There is still a tendency of the adhesion force to increase with reduced radius, but a clear confirmation of the linear relation between adhesion and reduced radius is not possible from these data. Nevertheless, the colloidal probe method can yield information such as the average adhesion force.

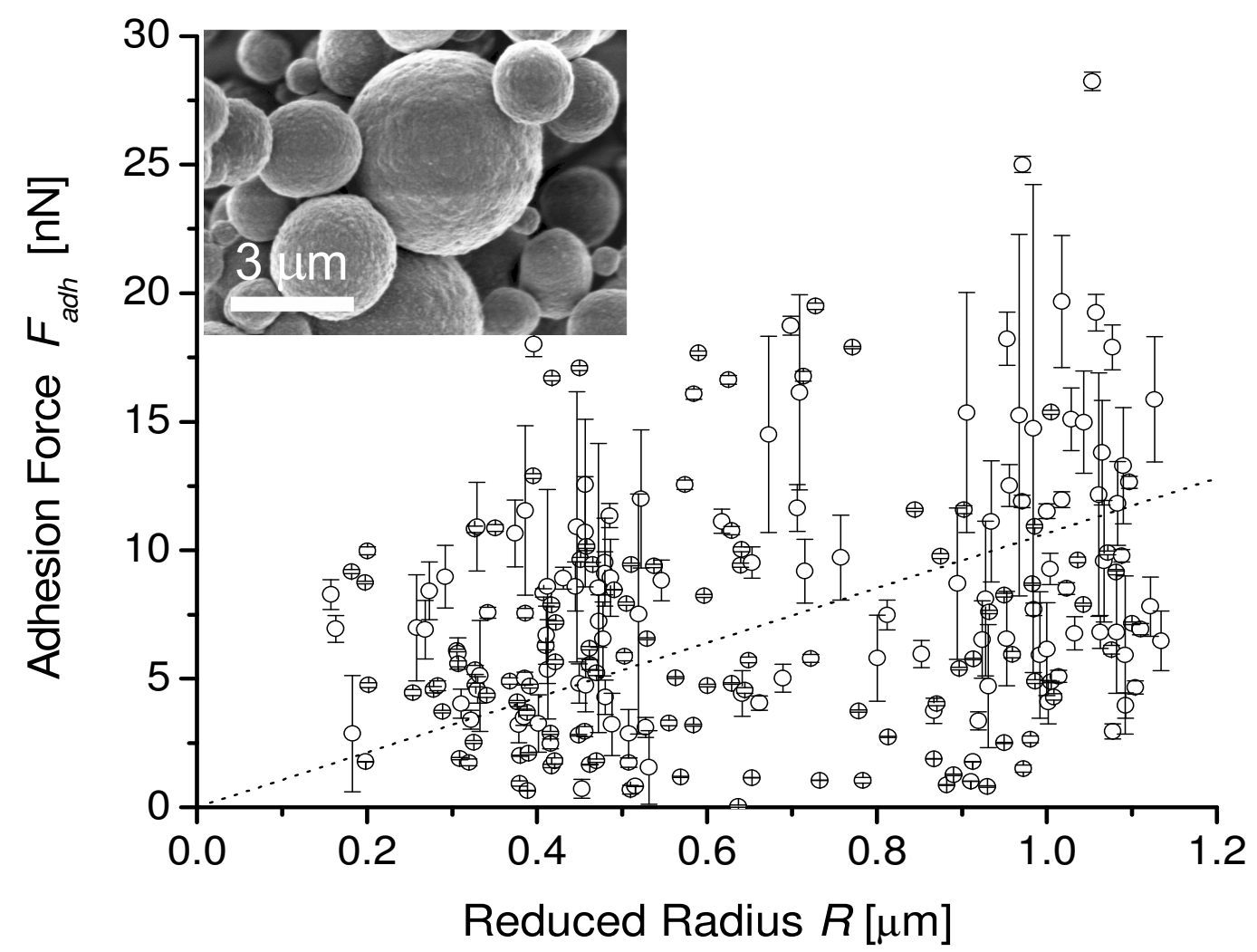

Fig. 5: Adhesion force between CIP particles plotted against the reduced radius of the particles. The much higher surface roughness, as compared to the silica particles (as apparent form the scanning electron micrograph shown in the inset), leads to a much larger variation of the adhesion forces. 

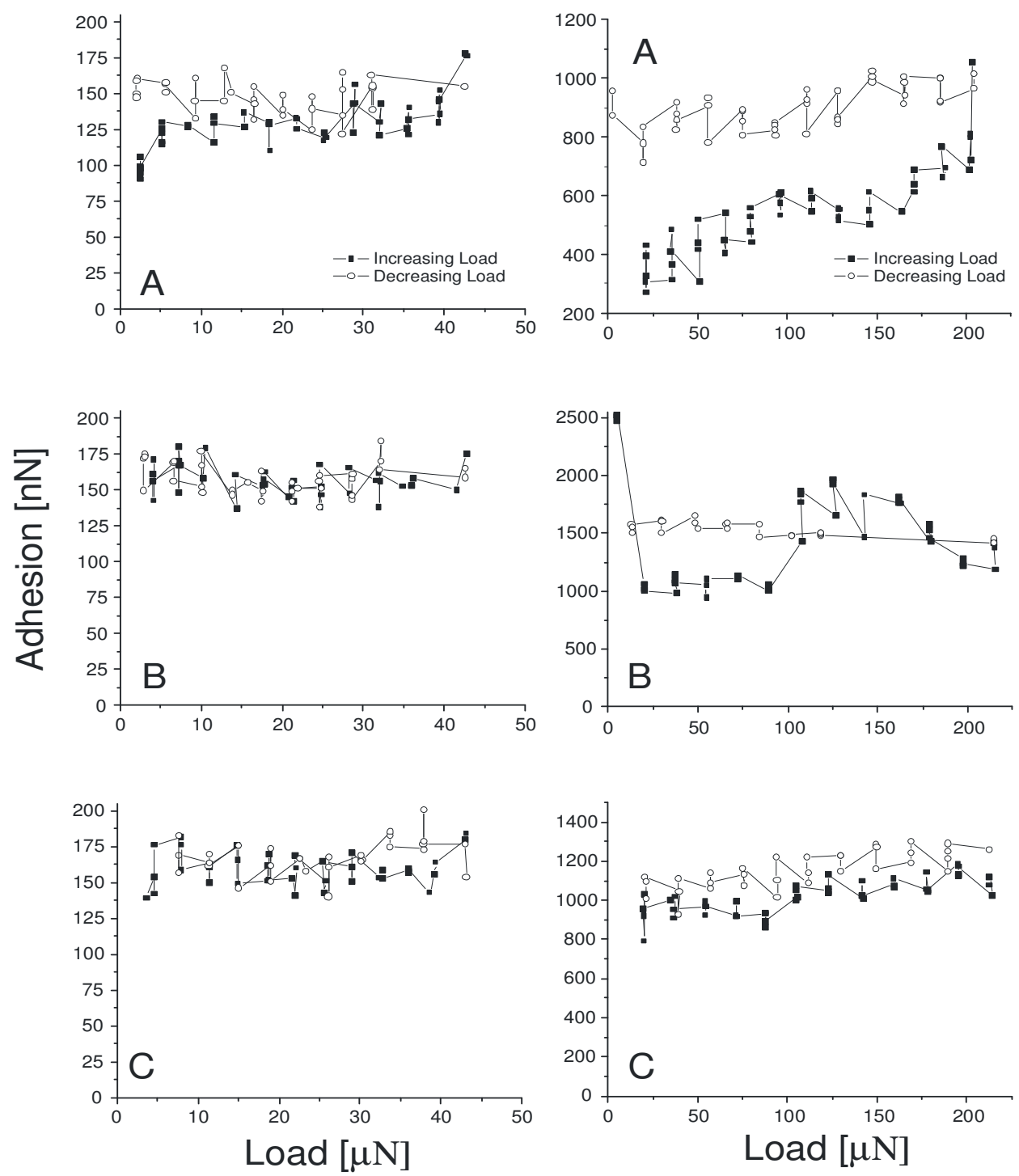

Fig. 6: Adhesion forces between two particles and a silicon wafer plotted against the applied load for a small (left, $R=3.0 \mu \mathrm{m}$ ) and a larger (right, $R=8.3 \mu \mathrm{m}$ ) particle. Plots A show the first load-unload cycle, where the load was stepwise increased (solid squares) to the maximum value and then decreased (open circles). An increase of the loading force from 2.5 to $43 \mu \mathrm{N}$ for the smaller particle (left) leads to an increase in adhesion of about $50 \%$. For the second particle (right) the adhesion increased by $160 \%$, while the load was increased from $21 \mu \mathrm{N}$ to $204 \mu \mathrm{N}$. In subsequent cycles $\mathrm{B}$ and $\mathrm{C}$, the load had no significant/systematic influence on the adhesion force, indicating that the plastic deformation of the particle mainly takes place in the first cycle.

In Fig. 6 the relation between adhesion force and loading force is plotted for two different particles which were mounted on cantilevers with spring constants of $3.8 \mathrm{~N} / \mathrm{m}$ (left) and 18.6 
$\mathrm{N} / \mathrm{m}$ (right), respectively. In each of the six plots a series of adhesion force measurements at the same spot on the silicon wafer are shown, where the load was first increased (solid squares) and then decreased (open circles). Data in the plots denoted with A are from the first cycle, plots B and C are from consecutive cycles taken at other spots on the silicon wafer, but from the same spot on the particle. For the smaller particle with the softer cantilever, an increase of about $50 \%$ in adhesion is observed (plot A, left) when the loading force is increased by a factor of 17 , from 2.5 to $43 \mu \mathrm{N}$. This increased adhesion level remains even when the loading force is reduced again. In the subsequent series (plots B and C, left) the adhesion does not change any more. For the larger particle with the stiffer cantilever, which allowed higher loading forces, adhesion increased by about $160 \%$ during the first cycle when increasing the loading force by a factor of 10 , from 21 to $204 \mu \mathrm{N}$ (plot A, right). For the third series (plot $\mathrm{C}$, right) no significant change in adhesion with load is observed and the value is about the same as the maximum reached during the first series. The second series (plot B, right) with this particle shows strong fluctuations, which might indicate a waver-surface irregularity at this spot.

\subsection{Friction force measurements}

Macroscopic sliding friction is commonly described by Amontons' equation (2) that states that the friction force $f_{T}$ is proportional to the sum of the normal (loading) force $f_{N}$ and the adhesion force $f_{0}$ and does not depend on the (apparent) contact area of the macroscopic bodies:

$f_{T}=\mu\left(f_{N}+f_{0}\right)$

For a single micro-contact, however, one expects that the friction force is given by:

$f_{T}=\tau A$

where $A$ is the true contact area of the micro-contact and $\tau$ is its shear strength, both of which are coupled with the normal (confinement) force. Discussing the details of the local stresses in the contact area is far beyond the scope of this paper. 
For the friction measurements, silica spheres with a radius of $2.5 \mu \mathrm{m}$ were used as colloidal probe. The sliding friction of these spheres on a silicon wafer was measured as a function of the loading force (Ecke 2001). Fig. 7 shows the dependence of the friction force on load for a single micro-contact (negative values of the loading force correspond to an applied pulling force to overcome the particle adhesion). Solid symbols correspond to the results for five different silica spheres on the native silicon wafer. The open symbols represent experiments where both surfaces have been treated with hexamethyldisilazane before the experiment. The reduction of adhesion and the lubricating effect of the silanization are clearly visible. The data have been fitted according to Eq. (3), assuming a constant shear strength and setting $A=\pi r_{c}{ }^{2}$, where $r_{c}$ is the radius of the contact area. The solid lines correspond to a fit where $r_{c}$ was calculated using the JKR theory, the dashed lines resulted from the calculation with the DMT theory. For both types of surfaces the JKR model fits better than the DMT model.

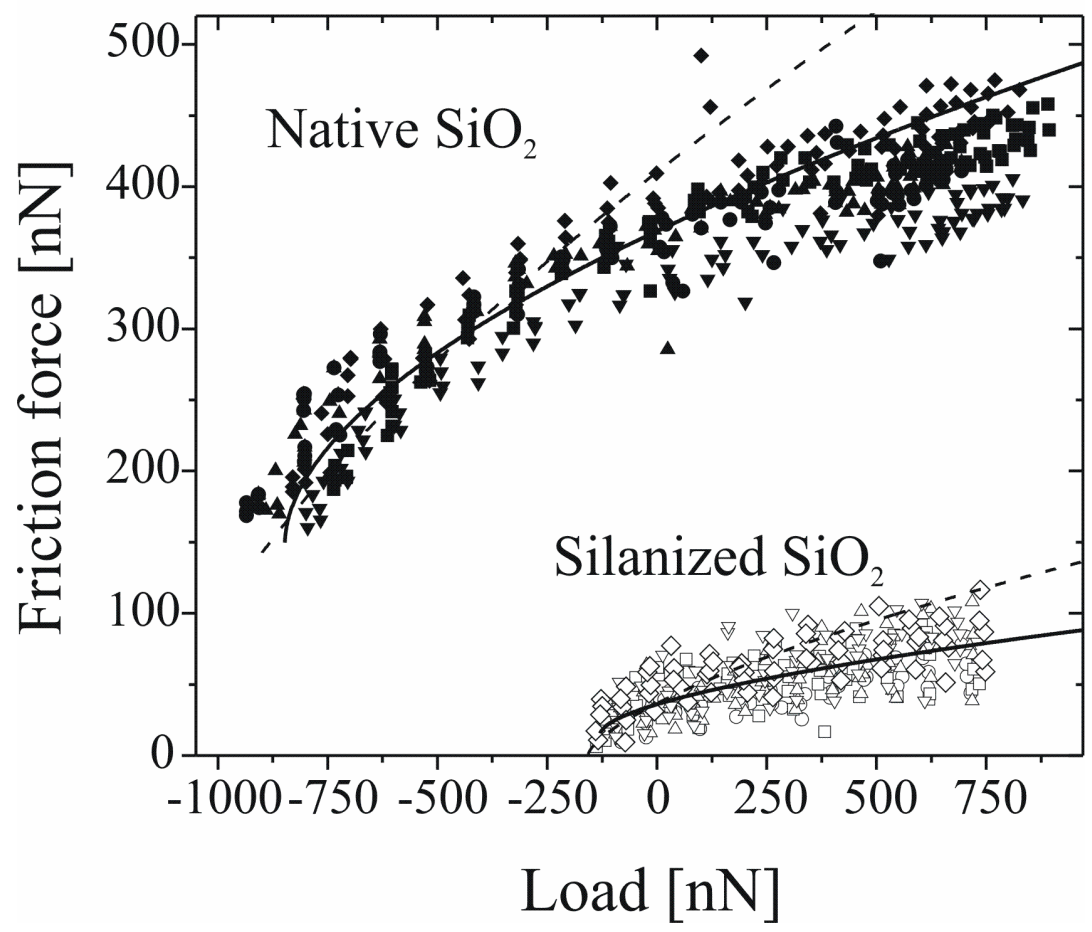

Fig. 7: Dependence of the friction force on the applied normal load for a single micro-contact. Solid symbols represent data from five different silica particles $(\mathrm{d} \approx 2.5 \mu \mathrm{m})$ on a native silicon wafer. The open symbols are data from experiments where both surfaces had been silanated. Data were fitted using Eq. (3), calculating the contact area by either using the JKR (solid line) or DMT (dashed line) theory. 


\section{CONTACT FORCES BETWEEN SINGLE PARTICLES AND CONTACT CONSTITUTIVE MODELS}

In terms of particle technology, powder processing and handling, the consolidation and nonrapid flow of dry, ultrafine and cohesive powders (particle diameter $d<10 \mu \mathrm{m}$ ) can be related to load-history dependent adhesion forces at the particle contacts. After defining such a particle-contact force-displacement law below, a many-particle simulation model can be applied.

\subsection{Particle interactions and deformations}

Here we focus on a characteristic, soft contact of two isotropic, rigid, mono-disperse spherical particles with diameter $d$. For convenience, the particles keep their spherical shape, i.e., they are rigid, and the (real) deformations at the contact are described by a (soft) forcedisplacement relation. In the simplest model, the soft contact force is a linear elastic and smooth function of the overlap $h_{K}$ of the particles, see Fig. 8 a). This soft or compliant contact displacement/deformation is of the order of nano-meters and thus can be assumed small $h_{K} / d<<1$. On the other hand it is large enough such that the contact area (and the deformed volume) consists of a representative number of molecules. Isotropy of the particles can be assumed if in powder processing, the particles are manufactured from uniform material in the bulk phase, without direction dependent processes involved.

\subsection{Particle contact constitutive models}

Fig. 8 shows a selection of particle contact constitutive models, the main constituent of a particulate many-particle system. The simple linear and non-linear elastic models are generalized to include plastic deformations and load-history dependent attractive forces. Considering various of these models, for a survey see Tomas (2003), one can formulate a more general contact model for time and rate dependent visco-elastic, plastic, visco-plastic, adhesion and dissipative behaviour (Tomas 1999, 2000, 2003). 
a) linear elastic

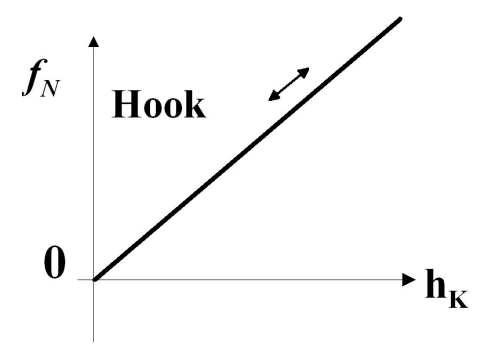

c) linear plastic, nonlinear elastic, dissipative, adhesion

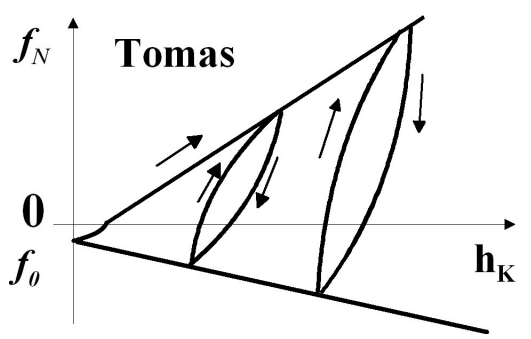

b) nonlinear elastic

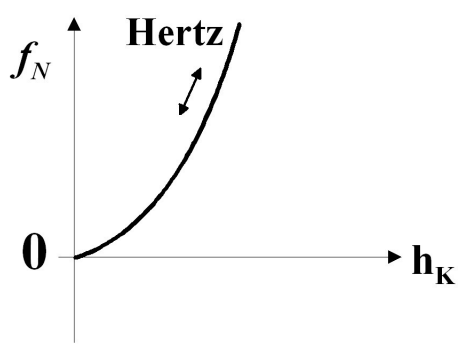

d) linear plastic, dissipative, adhesion

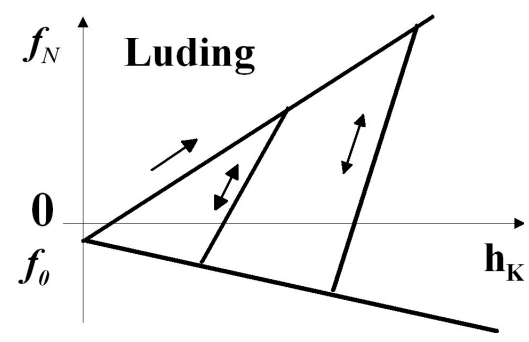

Fig. 8: Constitutive models of contact forces as function of deformation, in normal direction.

Positive deformation $h_{K}$ means compression, and positive (negative) force means repulsion (attraction). The most basic models for linear and non-linear elastic, reversible behaviour are based on Hook and Hertz (1882), respectively. These have been generalized by Tomas to include elastic-plastic contact flattening (Tomas 2001). Luding (2001, 2003, 2007) has simplified the model to a piecewise linear one, in order to allow a simpler implementation and more efficient numerical calculations. After the non-linear adhesion normal force model is applied, a micro-macro transition can be applied to obtain the elastic-plastic yield limits of a continuum (steady-state yield locus, instantaneous yield locus and consolidation yield locus) with the three flow parameters $\varphi_{i}, \varphi_{s t}$ and $\sigma_{0}$ (internal angle of friction, stationary angle of friction and isostatic tensile strength, respectively). This will be later discussed in more detail in Chapter 7.

Next we will discuss the constitutive models of Tomas (Fig. 9) and Luding (Fig. 10). The former is more elaborate and can be used as the basis for a description of the contact behaviour of cohesive particles, while the latter is applied in the many particle simulations below. 
In the framework of a mean-field approach, consider the contact of two particles as the basic element of a particle-packing under static load. If the particles are separated, very short ranged van der Waals forces are active. This is the physical reason for the point contact $\left(h_{\mathrm{K}}=0\right)$ adhesion force $f_{0}$, which itself can lead to some contact flattening/deformation, but will not be discussed in more detail here. In the case of compression $\left(h_{\mathrm{K}}>0\right)$, the yield limits of cohesive powders can be derived from physics/contact mechanics. For very small forces, an elastic contact flattening, as described by Hertz (1882), is obtained (Fig. 9).

If the normal load $f_{N}$ increases, first the yield point $Y$ is reached, where the deformation merges into the linear plastic flow regime, before the maximal normal force is reached at point $U$. The slope of the plastic flow line is the measure for the elastic-plastic contact stiffness. Lower slopes mean plastically soft or compliant contact behaviour, and a higher slope means stiffer contact behaviour. Viewed more closely, a confined plastic deformation field is created inside the circular contact area after loading. Above the (local) yield limit, the elastic domain resembles an annular zone enclosing the plastically deformed contact centre (Tomas 2001).

In this model it is assumed that unloading causes the elastic contribution to recover along a Hertz-type curve until the neutral point $E$ with zero force is reached. Further unloading requires a tension force, and eventually, the contact fails when the maximum tensile force is reached, i.e., the line with negative slope, at point $A$. The higher the slope, the more compliant is the contact behaviour and the higher could be the adhesion force of the particle contact. A lower slope corresponds to a stiff contact with almost constant adhesion force $f_{0}$. However, between the two limiting lines (two different un-/reloading paths are indicated in Fig. 8), the contact can still be reloaded. The lens-shaped area between the reloading and unloading curves is a measure for the energy dissipation during one cycle (Tomas 2001). Therefore, our contact shows hysteresis and, in the case of dynamic un-/re-loading cycles (oscillations), the energy-loss as a response does not depend upon an applied viscous, velocity-dependent force. The functional behaviour of the un-/re-loading cycle as well as the elastic-plastic yielding was measured and confirmed experimentally for small granulates with the diameter of $\sim 1 \mathrm{~mm}$ (Antonyuk 2005). However, the functional behaviour for tension, due to the van der Waals forces of realistic contacts of fine particles with complicated geometry, is still not experimentally validated. 


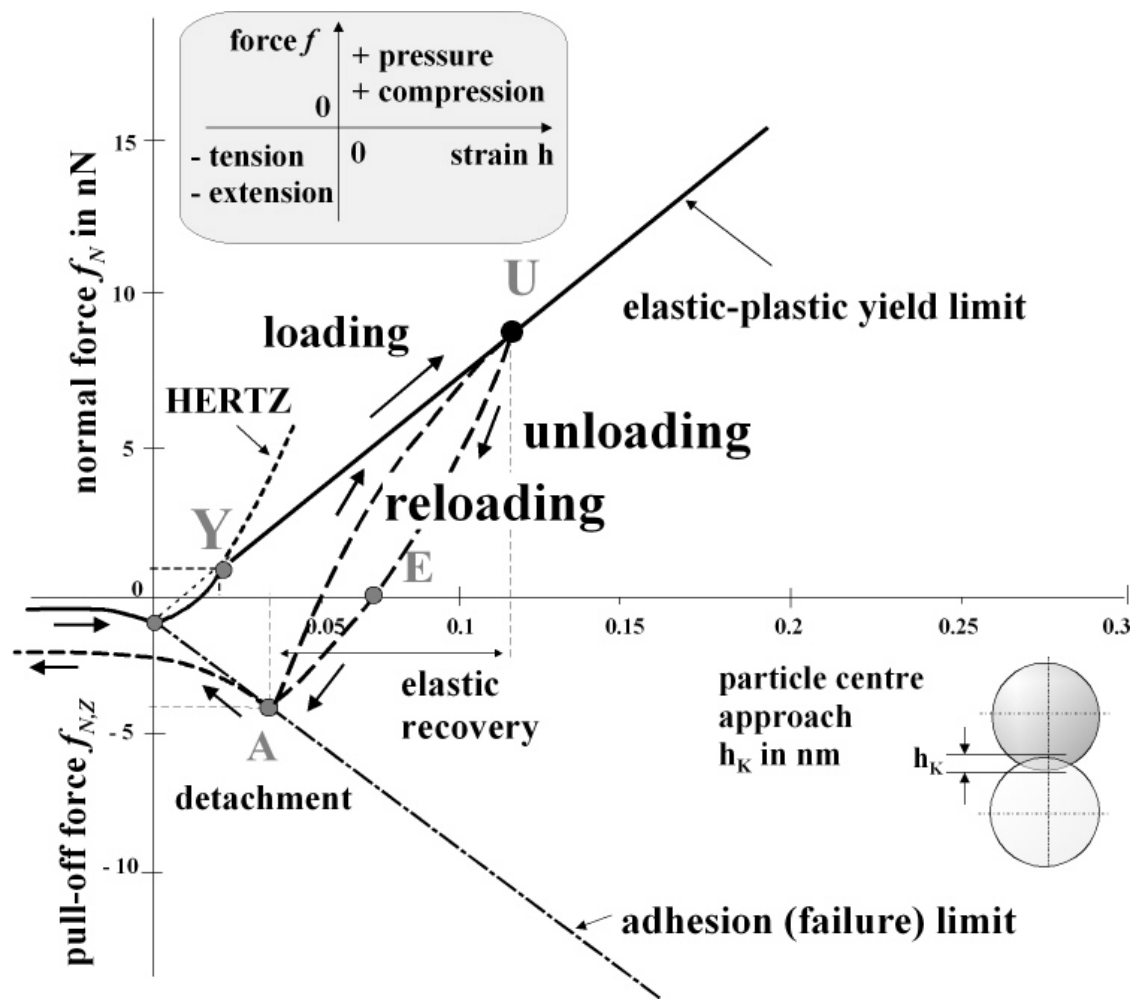

Fig. 9: Characteristic contact force as function of displacement (overlap) for $\mathrm{TiO}_{2}$

$\left(\mathrm{d}_{50}=0.61 \mu \mathrm{m}\right.$, moisture $\left.\mathrm{X}_{\mathrm{W}}=0.4 \%\right)$ - non-linear elastic, linear plastic, adhesive, dissipative contact model

Since this realistic and rather complicated contact model is not only very computer time consuming when implemented in the simulation model, and there is also a lack of information about the exact shape of the curves, we use the simplified contact law developed by Luding (2001, 2003, 2005, 2007), see Fig. 10: the Hertzian, elastic regime is completely neglected, but the limiting (linear) plastic yield and maximal adhesion limit-lines are kept. The hysteretic, lense-shaped un-/re-loading branches are replaced by a linear, elastic, reversible line with the slope increasing with the previously experienced maximal overlap. In order to include an energy-loss mechanism into this model that also works for small, slow deformations, a simple velocity-dependent dashpot is added. 


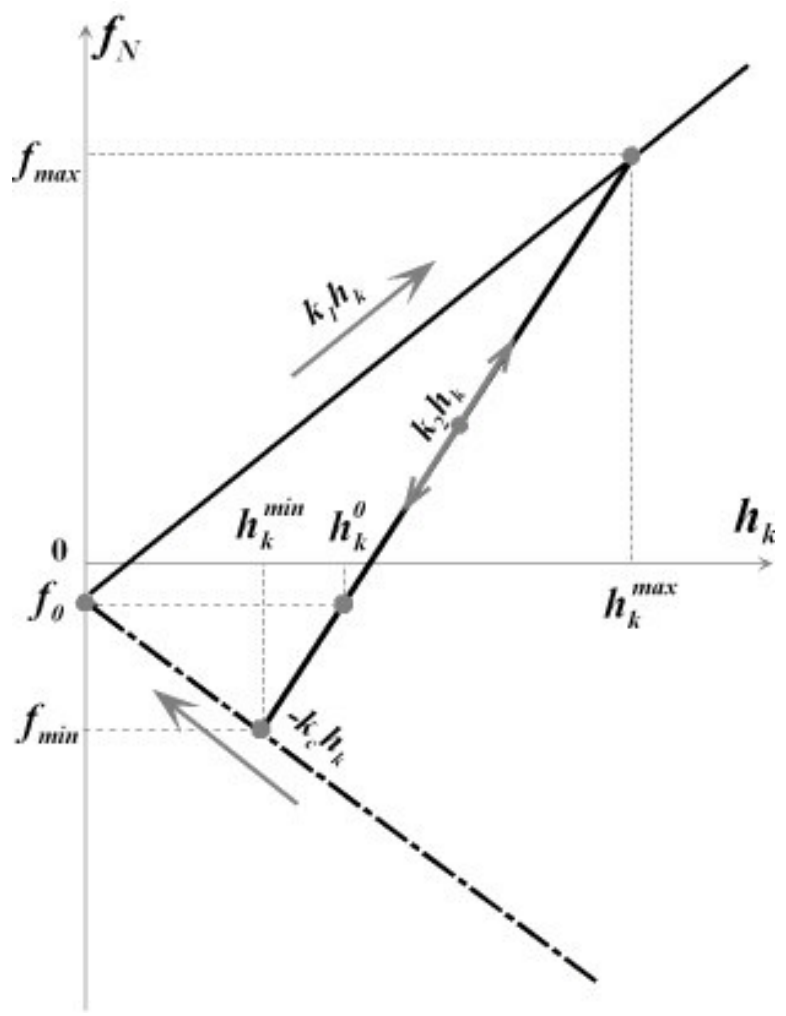

Fig. 10: Simplified, linearized force displacement law for adhesive, elasto-plastic contacts according to Fig. 9, as applied in the DEM simulations.

During loading, the repulsive force increases along the linear plastic yield limit, with stiffness $k_{1}$, that takes care of a "perfect" plastic (i.e. neglecting elastic) repulsion. Plastic deformation at the contact level is introduced by a linear spring with a larger stiffness $k_{2}$ for unloading and reloading, so that the stiffness increases due to plastic contact deformation/flattening (Luding 2007). (In the simulations presented in this paper, for the sake of simplicity, $k_{2}$ is assumed constant, with $k_{2}>k_{1}$.) The variable adhesion force (or "cohesion" on a microlevel) between the particles comes into the model by an "adhesion stiffness" $k_{\mathrm{c}}$, which defines the adhesion (failure) limit. This allows for attractive forces up to a maximum pull-off force $f_{\min }$ (per absolute value). Cast into an equation, the normal force on a particle is:

$f_{N}= \begin{cases}k_{1} h_{k}-f_{0} & \text { for loading } \\ k_{2}\left(h_{k}-h_{k}^{0}\right)-f_{0} & \text { for un-/ reloading } \\ -k_{c} h_{k}-f_{0} & \text { for unloading }\end{cases}$ 
The two contact models are compared in Table 1, with $r_{1,2}$ the median particle radius (characteristic radius of the contact surface curvature), $p_{f}$ the plastic micro-yield strength of the particle contact, $\kappa_{A}$ the elastic-plastic contact area coefficient, $\kappa_{p}$ the plastic repulsion coefficient, $E^{*}$ the averaged modulus of elasticity, $h_{k}^{\min }$ and $h_{k}^{\max }$ minimal and maximal magnitudes of overlap or centre approach during one cycle, $p_{V d W}$ the attractive van der Waals pressure, and $a_{F=0}$ the minimum centre separation for molecular force equilibrium.

Table 1: Comparison of the two considered contact models

\begin{tabular}{|l|c|c|}
\hline & Tomas & Luding \\
\hline Loading & $\pi r_{1,2} p_{f}\left(\kappa_{A}-\kappa_{p}\right) h_{k}-f_{0}$ & $k_{1} h_{k}-f_{0}$ \\
\hline Unloading & $\frac{2}{3} E * \sqrt{r_{1,2}\left(h_{k}-h_{k}^{\text {min }}\right)^{3}}-\pi r_{1,2} \kappa_{p} p_{f} h_{k}^{\text {min }}-f_{0}\left(^{*}\right)$ & $k_{2}\left(h_{k}-h_{k}^{0}\right)-f_{0}$ \\
\hline Reloading & $-\frac{2}{3} E^{*} \sqrt{r_{1,2}\left(h_{k}^{\text {max }}-h_{k}\right)^{3}}+\pi p_{f} r_{1,2}\left(\kappa_{A}-\kappa_{p}\right) h_{k}^{\text {max }}-f_{0}$ & $k_{2}\left(h_{k}-h_{k}^{0}\right)-f_{0}$ \\
\hline Adhesion & $-\pi r_{1,2} p_{V d W} h_{k}-f_{0}$ & $-k_{c} h_{k}-f_{0}$ \\
\hline limit & & \\
\hline
\end{tabular}

(*) $h_{k}^{\min }$ has to be obtained iteratively, depending on $h_{k}^{\max }$, as the solution of the cubical equation describing an elastic, Hertz-type unloading curve.

The unloading and reloading branches of the two models can not be directly related to each other. The limit lines, on the other hand, are $k_{1}=\pi r_{1,2} p_{f}\left(\kappa_{A}-\kappa_{p}\right)$ and $k_{c}=\pi r_{1,2} p_{V d W}$, and the maximum adhesion force, as can be obtained experimentally from AFM experiments, is the adhesion limit, evaluated at $h_{k}^{\text {min }}$.

\subsection{Load dependent maximum adhesion force}

In order to oppose/compare the two models above, we express the contact adhesion force, $f_{H}$, in terms of the applied load $f_{N}$ (note that $f_{H}$ is now positive for convenience, i.e., the absolute value of $f_{\min }$ in Fig. 10, for details see Tomas 2002). The value of $E^{*}$ is taken from tables for material properties, whereas $p_{f}, \kappa$, and $\kappa_{p}$ are back-calculated from shear experiments (Tomas 2002). 
Taking into account the curvature of the unloading line U-A in Fig. 9 (similar to a Hertz curve), the non-linear adhesion force (Tomas 2002) is derived iteratively, with some arbitrary starting value $f_{H}^{0}$ :

$f_{H}^{i+1}=f_{0}+\kappa\left(f_{N}+f_{0}\right)-\pi r_{1,2}^{2} \kappa_{p} p_{f}\left[\frac{3\left(f_{N}+f_{0}\right)}{2 r_{1,2}^{2} E^{*}}\left(1+\frac{f_{H}^{i}-f_{0}}{f_{N}+f_{0}}\right)\right]^{2 / 3}$

In first approximation (when $f_{H}^{0}=-f_{N}$ is used as starting value), the simplified (linear) adhesion force looks like:

$f_{H}^{1}=(1+\kappa) f_{0}+\kappa f_{N}$,

practically neglecting the last term in Eq. (5).

For the Luding model, the characteristic pull-off or adhesion force is also a linear function of the compression or preconsolidation force $f_{N}$

$f_{H, L u}=\frac{1+k_{c} / k_{1}}{1+k_{c} / k_{2}} f_{0}+\frac{k_{2} / k_{1}-1}{k_{2} / k_{c}+1} f_{N}=\left(1+\kappa_{L}\right) f_{0}+\kappa_{L} f_{N}$,

with $\kappa_{L}=\kappa \frac{h_{k}^{\min }}{h_{k}^{\max }}=\frac{k_{2} / k_{1}-1}{k_{2} / k_{c}+1}$.

Fig. 11 shows the quantitative difference between the above mentioned models for the adhesion force in dependence on the normal force, for the example of shear tests with limestone, $\mathrm{CaCO}_{3}, d_{50} \approx 1.2 \mu \mathrm{m}$, humidity $X_{W}=0.5 \%$. The parameters used are $p_{f}=350 \mathrm{MPa}$, $\kappa=0.223, \kappa_{p}=0.152$, and $E^{*}=150 \mathrm{kN} / \mathrm{mm}^{2}$; in Eq. (7); furthermore, $\kappa_{L}=\frac{2}{3} \kappa$ was used leading to best agreement for moderate forces. 


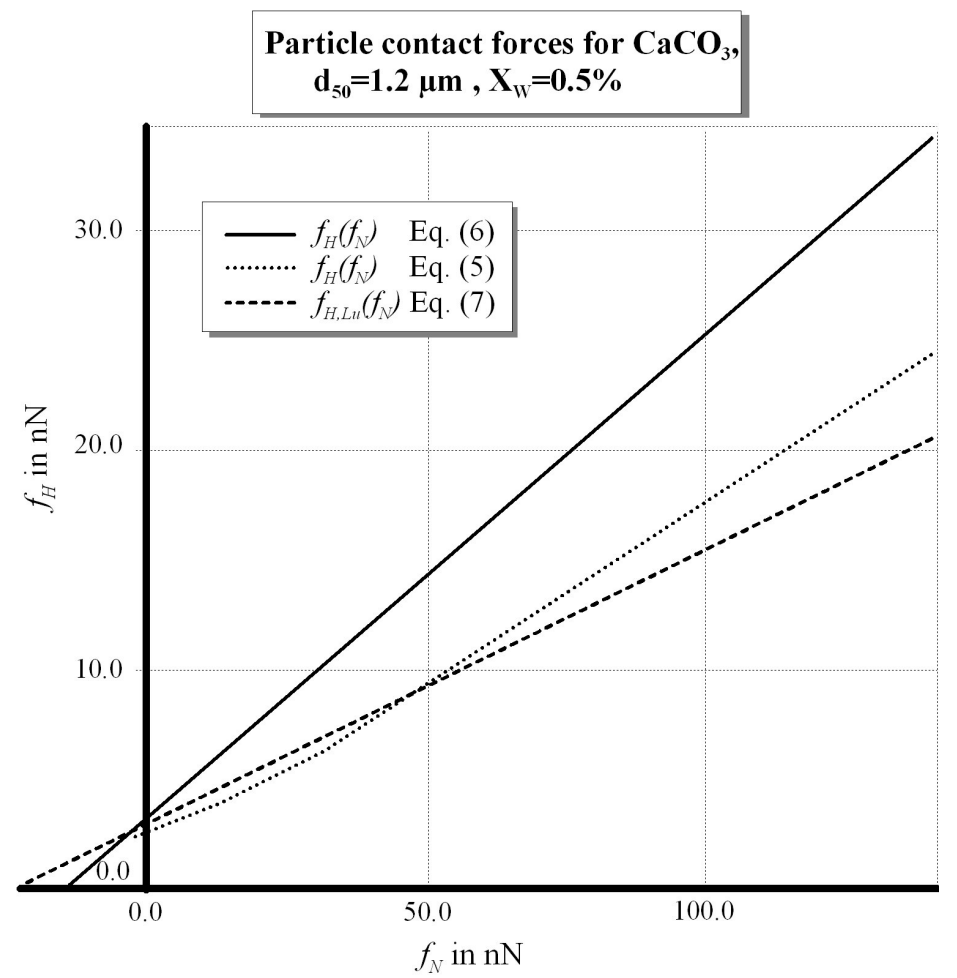

Fig. 11: Characteristic adhesion force vs. normal force functions for limestone, back calculated from shear tests: comparison of the linear Tomas model, Eq. (6), the non-linear Tomas model, Eq. (5), and the (linear) Luding model, Eq. (7)

The first observation from Fig. 11 is that the linearized form of the Tomas model is leading to considerably larger adhesion forces as the non-linear version - for which the back-calculation of parameters was actually performed. This means, with other words, that the non-linear term in Eq. (5), is important and cannot be neglected. The second observation is that the Luding model leads to comparable adhesion forces as predicted by Eq. (5), if $\kappa_{L}=\frac{2}{3} \kappa$ is used. This allows to adjust $k_{\mathfrak{c}}$, since it is the only free microscopic model parameter $\left(k_{2}\right.$ is responsible for the contact stiffness, and the ratio $k_{1} / k_{2}$ determines the amount of plastic deformation at the contact).

\subsection{Tangential force of a load dependent adhesion contact}

The tangential force involves dissipation due to Coulomb friction, but also some tangential elasticity that allows for stick-slip behaviour on the contact level (Luding 2001; Luding 2003; Thornton 2000; Tomas 2001; Tomas 2003). 
In the static case, the tangential force is coupled to the normal force via Coulombs law, i.e. $f_{T} \leq \mu_{s} f_{N}$, and for the limit sliding case one has dynamic friction with $f_{T}=\mu_{d} f_{N}$. The dynamic and the static friction coefficients follow, in general, the relation $\mu_{d} \leq \mu_{s}$. However, for the following simulations, we will apply $\mu=\mu_{d}=\mu_{s}$. From the modelling point of view, the static case requires an elastic spring in order to allow for a restoring force, i.e., a non-zero remaining tangential force in static equilibrium due to activated Coulomb friction, as first implemented by Cundall \& Strack (1979).

The tangential force-displacement relation can be expressed by a linear elastic contribution for the no-slip region within the contact area according to Hooke's law (Tomas, 2003):

$f_{T}=4 G^{*} r_{K} \delta$

with $G^{*}$ the shear modulus of the contact, $r_{K}$ the contact radius and $\delta$ the contact displacement in shear direction (plane).

The contact radius $r_{K}$ depends on the actual normal force $f_{N}$, the adhesion force $f_{H}$, and the ratio $G * / E *$ (where $E *$ is the contact modulus of elasticity), :

$f_{T}=4 G *\left[\frac{3 \cdot r_{1,2} \cdot\left(f_{N}+f_{H}\right)}{2 \cdot E^{*}}\right]^{1 / 3} \delta$,

so that the initial tangential stiffness $k_{T, o}\left(f_{N}\right)$ increases with increasing preconsolidation $f_{H}$, see Fig. 12 (Tomas, 2003). 


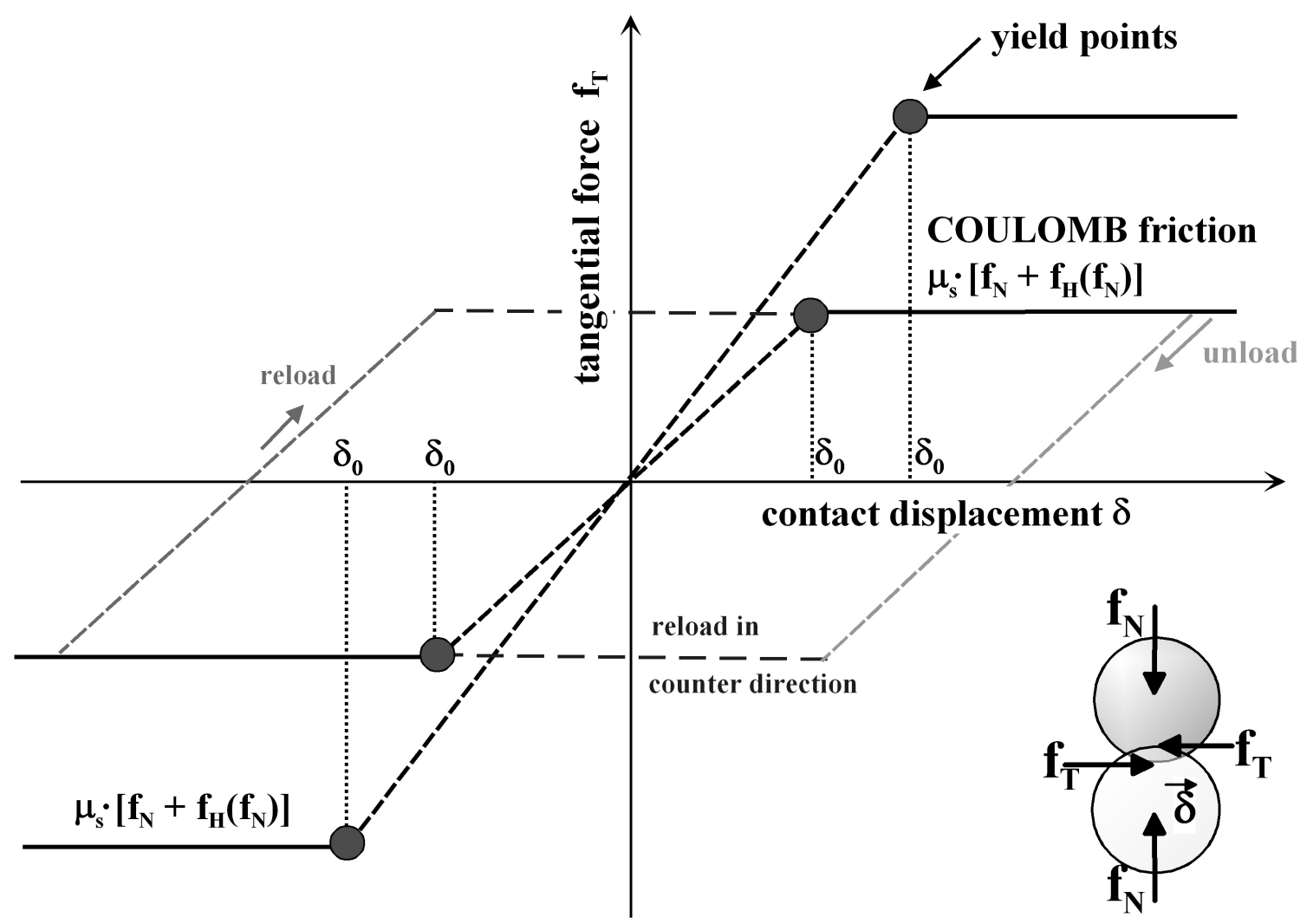

Fig. 12: Tangential force-displacement diagram. The elastic range is limited by the tangential displacement $\delta_{0}$, which is increasing with increasing normal load. At the yield point(s) the elastic behaviour is transmitted into the frictional, sliding behaviour, as shown by the constant tangential force lines, Eq. (10).

The contribution of Coulomb friction is described by the coefficient of internal friction (static friction) $\mu_{s}$ and depends on the contact consolidation, i.e., on the given normal force $f_{N}$ and the variable adhesion force $f_{H}\left(f_{N}\right)$ as well:

$f_{T}=\mu_{s} \cdot\left[f_{N}+f_{H}\left(f_{N}\right)\right]=\mu_{s} \cdot(1+\kappa) \cdot\left(f_{0}+f_{N}\right)$

Using Eqs. (8) and (10) the transition between the elastic regime and incipient contact failure (sliding) is the friction limit of tangential displacement $\delta_{0}$, see the yield points in Fig. 12:

$\delta_{0}=\frac{\mu_{s} \cdot\left[f_{N}+f_{H}\left(f_{N}\right)\right]}{4 \cdot G^{*} \cdot r_{K}}$ 
For tangential displacements larger than the limit $\delta_{0}$, energy is dissipated by irreversible friction work.

\section{SIMULATION BACKGROUND}

\subsection{Discrete element method (DEM)}

One possibility to gain insight into the material behaviour of a granular packing, is to perform elementary tests in the laboratory. Here, we chose as alternative the simulation with the discrete element model (Oda, 2000; D’Addetta, 2002; Kruyt, 2001; Luding, 2001; Luding, 2004a; Tykhoniuk, 2004).

The discrete (or distinct) element solution scheme assumes each of its constituents as a separate entity. The mechanical behaviour of a system consisting of, in general, randomly shaped particles, can be simulated by a generalized particle dynamics model, similar to the widely used molecular dynamics. The particles in this type of model are a-priory treated independently and interact only at contact amongst each other or with the interfaces (walls). If the particles are assumed to be rigid spheres, and the behaviour of the contacts is characterized by a soft contact model, as described in Section 3.2, then the mechanical behaviour of such a system is completely described in terms of Newton's laws of motion for the translational and rotational degrees of freedom:

$m_{i} \frac{d^{2}}{d t^{2}} \vec{r}_{i}=\vec{f}_{i}+m_{i} \vec{g} \quad$ and $\quad I_{i} \frac{d}{d t} \vec{\omega}_{i}=\vec{t}_{i}$

with the gravitational acceleration $\vec{g}$, mass $m_{i}$ of the particle, its position $\vec{r}_{i}$, the total force $\vec{f}_{i}$, acting on it due to contacts with other particles or with the walls, its moment of inertia $I_{i}$ (for the sake of simplicity spheres are used in the following), its angular velocity $\vec{\omega}_{i}$, and the total torque $\vec{t}_{i}$.

The integration of Eq. (12) is performed with a fixed time step, $\Delta t \cong \frac{t_{c}}{100}=\frac{\pi}{100} \sqrt{\frac{m}{k}}$, where $t_{\mathrm{c}}$ is the typical duration of the collision of two particles, $m$ is the relevant (reduced) mass, and $k$ 
the corresponding elastic stiffness constant, see also the following subsection. The discrete element method is thus formulated as a finite difference scheme and works in principle similar to the time marching scheme of an explicit finite element solution. The way of assigning different properties to individual particles (elements) in DEM is much simpler than in the case of the finite element method. The detailed calculation procedure is given, for example, in Allen and Tildesley (1989) or in PFC2D (see the Manual of PFC2D).

\subsection{Time steps and contact duration times}

The numerical time step $\Delta t$ plays a vital role for the calculations and thus for the results. If the calculation time step is too large, the system may not be able to react to all the occurring dynamics: for example, two particles may cross each other if their relative speed is high and the time step is large, $\Delta t>d / v$, with diameter $d$ and speed $v$. This means also that, e.g., propagating waves are not properly transmitted from one particle to the next. Even for a static system with $v=0$, too large time step go ahead with an unphysical gain in kinetic energy due to numerical instability. On the other hand, if $\Delta t$ is too small, it unnecessarily consumes time for the calculations. With other words, the solution of the equations of motion is stable only if the integration time-step does not exceed a critical time-step much smaller than the minimum eigen-period of all the particle contacts. Especially in the case of strongly different contact properties (stiffness) and masses, a local time-step would be optimal. This is impractical, however, due to the large and constantly changing system and contact properties typically encountered.

An estimate for the time-step is given in the following.

The motion of a simple mass-spring system of a point mass $m$ and a spring with stiffness $k$, is governed by the differential equation $-k x=m \ddot{x}$, so that the eigen-period is given by

$$
T=2 \pi \sqrt{\frac{m}{k}},
$$

with $t_{\mathrm{c}}=T / 2$ is the typical duration of a contact. This time has to be properly resolved for numerical integration, see the definition of $\Delta t$ above. For different weighted masses and the same stiffness, the minimum mass must be taken into account, and for fixed mass, the 
maximum stiffness has to be used for estimating the time step. For a more detailed calculation, see Luding (1998).

When there are many particles, with different mass and stiffness, the smallest eigenperiod will occur when the masses are moving in a synchronized opposing mode. Similarly to the translational relative motion, the rotational degrees of freedom have an eigenperiod of the order of $t_{\text {rot }} \cong \pi \sqrt{\frac{q}{1+q} \frac{m}{k^{r o t}}}$,

where $k^{\text {rot }}$ is the effective rotational stiffness determined by the tangential springs in the contact model, and $q=0.4$ for spheres with radius $a$ and moment of inertia $I=q m a^{2}$. As a consequence, the tangential springs have typically to be chosen considerably weaker than the normal springs so that $t_{\text {rot }} \approx t_{c}$. If the tangential springs are too strong, the response time of the rotational degrees of freedom becomes too small and the time step would have to be reduced.

The next important time is the time information needs to propagate from one end of the

system to the other, which determines the relaxation time $t_{L} \cong \frac{l}{d} t_{c}=L t_{c}$ for a system with length $l$, particles with diameter $d$, and contact response time $t_{\mathrm{c}}$. The system response time can become quite large for large systems $L>>1$, requiring even longer simulation times $t_{\text {sim }}>>t_{L} \cong L t_{c} \cong 100 L \Delta t$ for quasi-static simulations as presented below.

\section{MODEL SYSTEMS}

In this section two different model systems are compared. The translational (Jenike) shear cell set-up is discussed in section 5.1 and the bi-axial box is introduced in section 5.2. Preparation and initial condition issues are discussed in section 5.3.

\subsection{Translational Shear Cell}

The classical translational shear cell set-up, developed by Jenike (1964), is modelled below. The system consists of two cylindrical parts that contain the powder and are sheared against each other. In order to limit the CPU-time, only a small two-dimensional (2D) slice from the real shear cell is simulated. Fig. 13 shows the set-up for the cell filled with model powder to a 
required porosity. The predefined normal stress $\sigma=F_{N} / A$ (the area of the cylinder $A$ is replaced by the length of the slice $d_{Z}$ in the 2D case) is applied on the upper cylinder top, which is free to move perpendicular to the cylinder ring walls. The top wall (shear lid) is stress controlled, i.e., when the reaction force $F_{N}$ changes because of some particles reorganize, the height of the shear lid is changed accordingly. The horizontal shear rate of the upper part of the cell is preset, i.e., the upper ring is strain driven. As direct response, the corresponding values of the reaction forces $F_{S}$ are obtained, which act on the lateral walls, and the corresponding shear stresses $\tau=F_{S} / A$ are calculated, to obtain the flow parameters of the simulated model powder. The shear rate applied here is about 1-4 $\mathrm{mm} / \mathrm{min}$ (similar to the one used in the Jenike shear cell in laboratory tests). During the simulation, the shear stress $\tau$ is recorded as function of the shear strain, for different normal stresses $\sigma$.

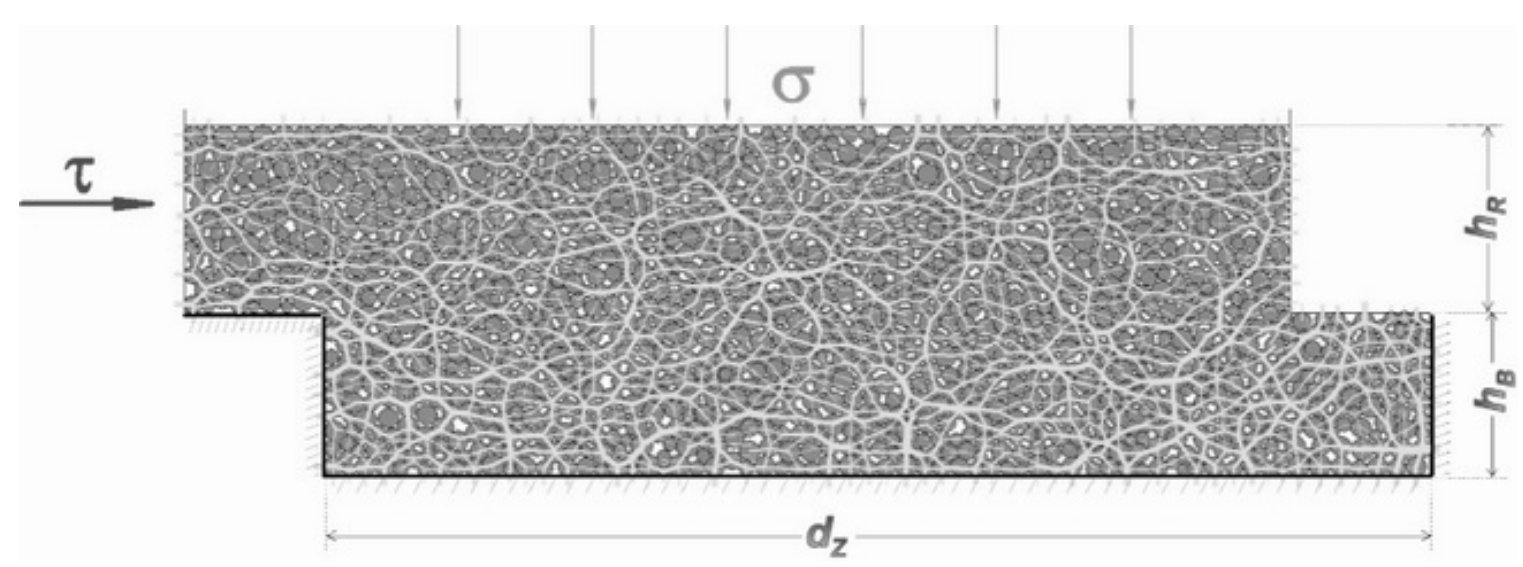

Fig. 13: The 2D-shear cell model system for the simulations (the thickness of the light-grey lines in the particle system indicates the (initial) contact forces)

\subsection{Biaxial box}

Besides the rather simple experimental realization of the Jenike-cell, it is quite inhomogeneous geometrically, and does not allow for a direct control of stress and strain. An alternative device is the bi-axial box where the powder is enclosed by plane walls. Besides simple compression tests, where all walls move in, shear can be realized, for example, by moving two opposing walls in and the perpendicular walls out.

Here, a two-dimensional biaxial box is used as the model system, see Fig. 14, where the left and bottom walls are fixed. Stress- or strain-controlled deformation is applied to the side- and top-walls, respectively. 

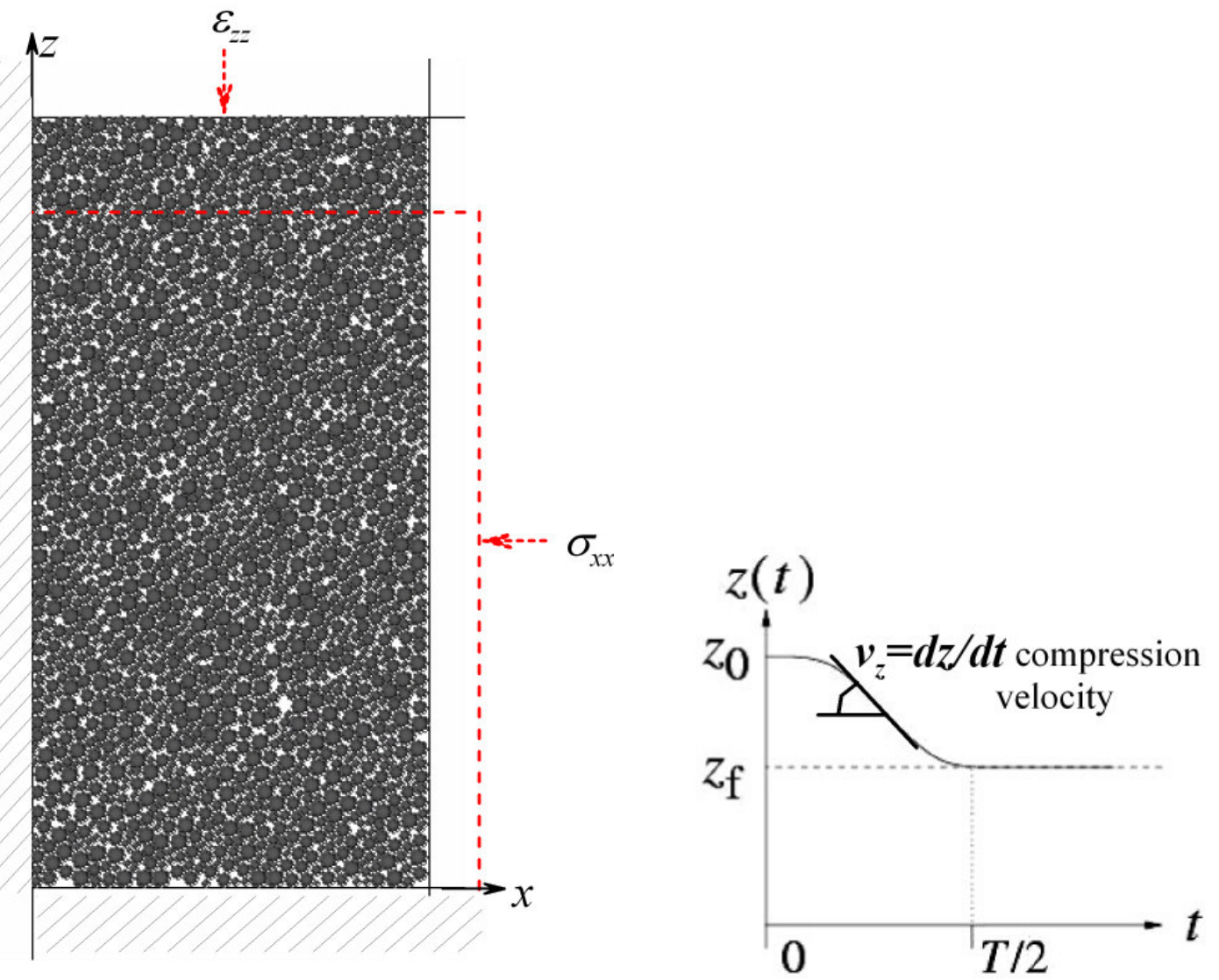

Fig. 14: The 2D-biaxial box model system for the simulations (Luding, 2001, 2003, 2004b)

In a typical simulation, the top wall is slowly shifted downwards, while the right wall moves, controlled by a constant stress $\sigma_{x x}$, responding on the forces exerted on it by the material in the box. The motion of the top wall follows a cosine function, in order to allow for a smooth start-up (at the height $z_{0}$ ) and finish (at $z_{f}$ ) of the motion so that shocks and inertia effects are reduced, however, the shape of the function is arbitrary as long as it is smooth and motion is slow.

As different from the Jenike set-up, here the major principal stress $\sigma_{z z}$ and the minor principal stress $\sigma_{x x}=$ const. are recorded as function of the vertical wall strain $\varepsilon_{z z}$. Before the results from both set-ups are related to each other in the next section, the initial sample preparation is discussed. 


\subsection{Initial conditions}

Initially, the particles are randomly distributed in a huge box, with rather low overall density. Then the box is compressed by defining an external pressure, $\sigma_{x x}=\sigma_{z z}=p$ in order to achieve an isotropic initial condition with kinetic energy much smaller than the potential energy stored in the contacts. Starting from this relaxed, isotropic configuration, the strains are applied to both model systems and the response of the system is examined, as quantified by stress and volumetric strain

$\varepsilon_{V}=\frac{V(t)-V_{0}}{V_{0}}$,

with $V(t)=A h(t)$ being the dynamic and $V_{0}=A h_{0}$ the initial volumes of the translational shear cell, while for the bi-axial box, one uses $V(t)=L_{x}(t) L_{y}(t)$ and $V_{0}=V(0)=L_{x}(0) L_{y}(0)$.

\section{REFERENCE SHEAR EXPERIMENTS}

In order to compare simulations with experiments quantitatively (the stresses as well as the volumetric strain values), laser position sensors are mounted on a standard translational shear cell (Fig. 15). The three triangulating laser displacement sensors allow to measure the dynamic change (movement and tilt) of the shear lid during the shear test, and thus the volumetric strain versus shear displacement. A typical experiment consists of a preconsolidation step (without tangential shear, but with twist or vibration to enhance the consolidation) at normal stress $\sigma_{N, 0}$, pre-shear steps with normal stress $\sigma_{\text {pre }}$, and the shear steps with lower normal stresses, from which eventually the yield stresses are obtained. A good consolidation is needed to over-consolidate the powder, so that the following shear tests can be performed within the very short strain path $(6 \mathrm{~mm})$ available in this set-up. The preshear is used to get into the critical state flow regime, so that the subsequent shear tests are always starting from well defined initial conditions. In the following some typical tests are reported. 


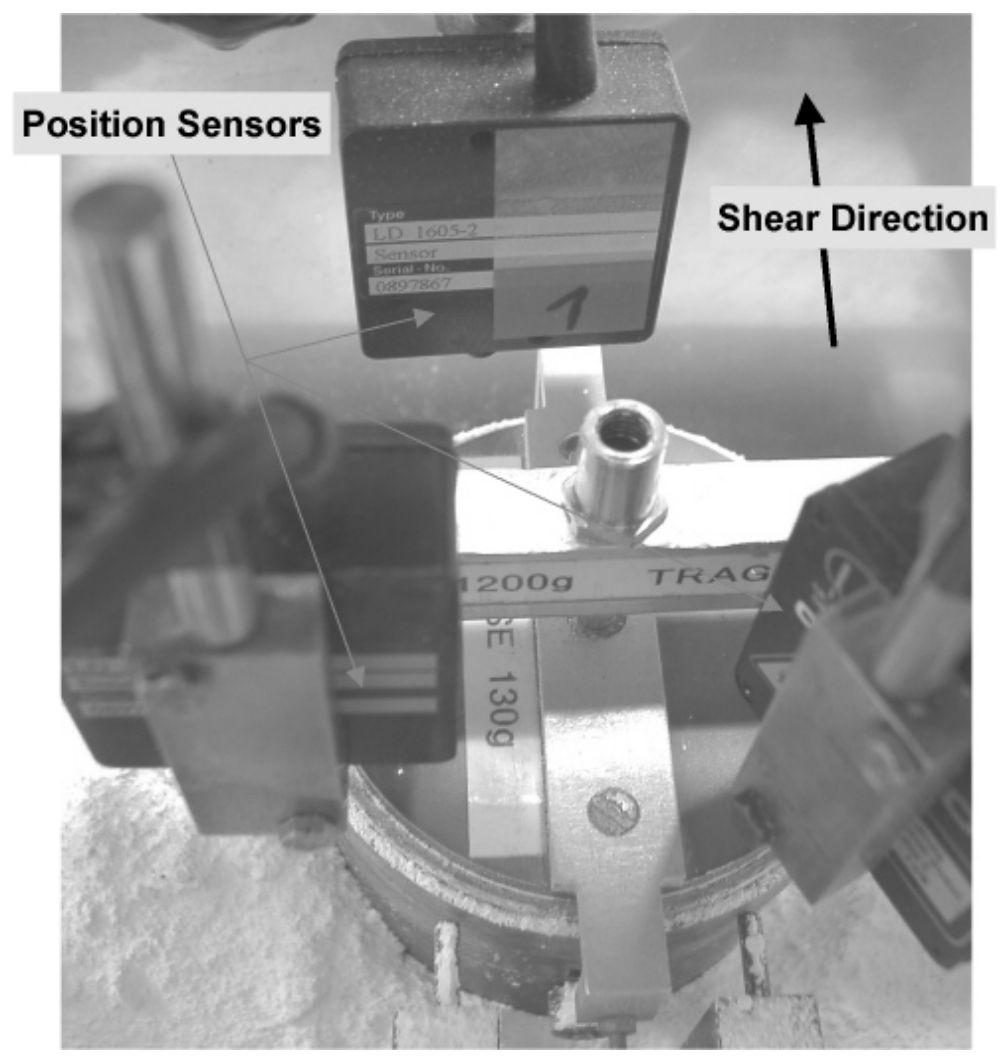

Fig. 15: Translational shear cell with shear lid height measurement by laser sensors

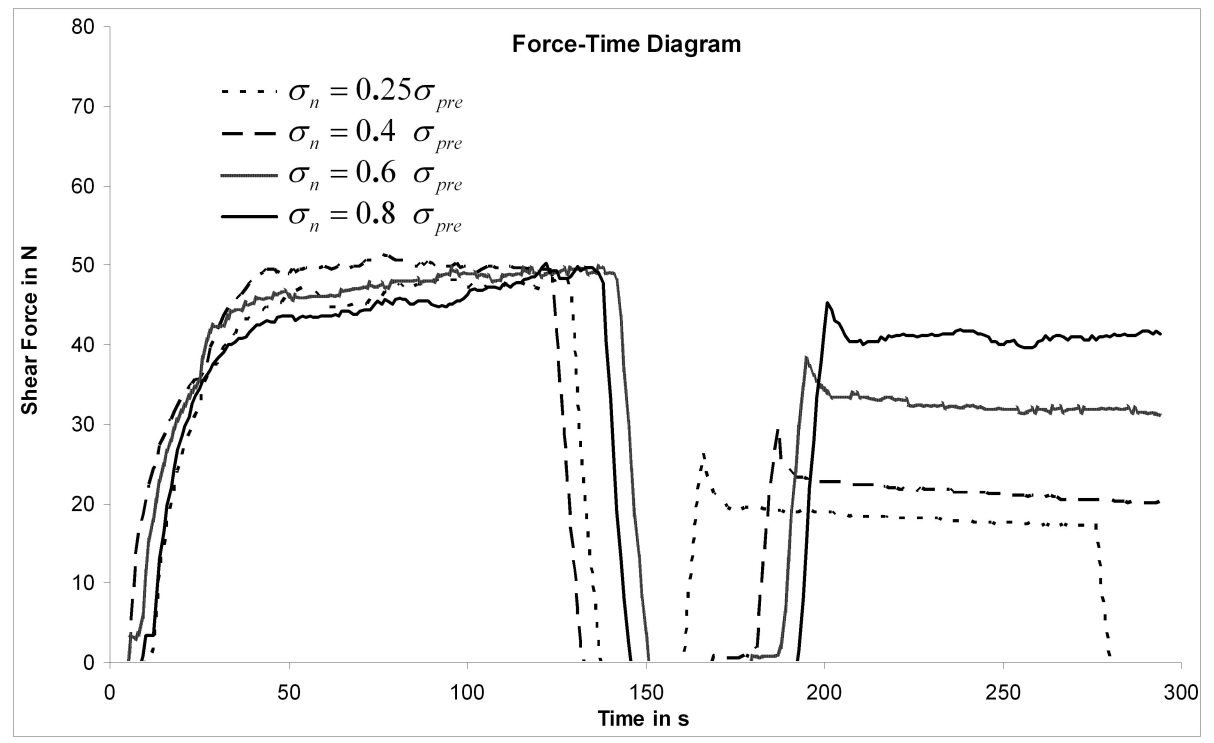




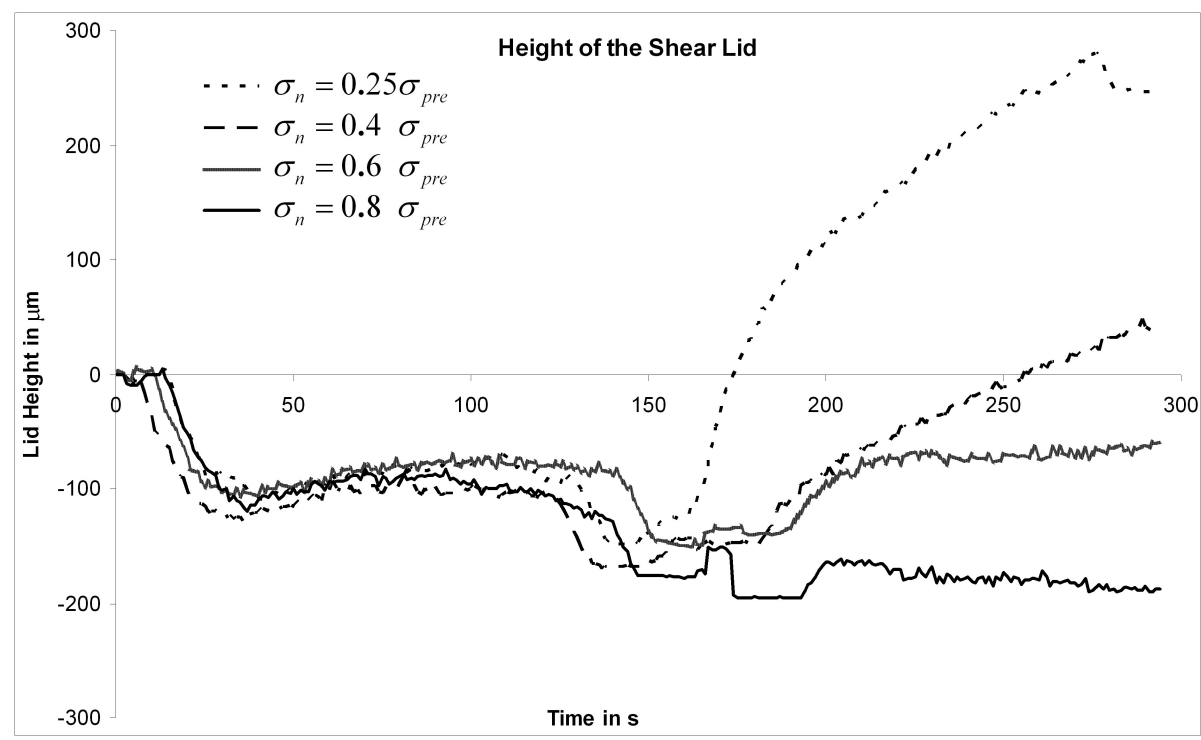

Fig. 16 (Top) Shear force measurements during shear tests (limestone powder)

(Bottom) Volumetric strain measurements during the same shear test

The tests were performed with a limestone powder sample with average particle diameter $d_{50}=1.2 \mu \mathrm{m}$, density $\rho_{s}=2740 \mathrm{~kg} / \mathrm{m}^{3}$, and a shear rate of $v_{s}=2 \mathrm{~mm} / \mathrm{min}$. Diameter of the cell is $95 \mathrm{~mm}$, height of the base ring $14.5 \mathrm{~mm}$ and height of the shear ring $16 \mathrm{~mm}$. Fig. 16 shows an example of the stress and volumetric strain measurements. The powder was preconsolidated with normal stress $\sigma_{N, 0}=16 \mathrm{kPa}$ and pre-sheared with the normal stress $\sigma_{\text {pre }}=8 \mathrm{kPa}$. The normal stress $\sigma_{n}$ during the shear procedure was taken proportional to $\sigma_{\text {pre }}$ with the factors $0.25,0.4,0.6$ and 0.8. Some compression (about $0.1 \mathrm{~mm}$, i.e., about 80 particle diameters!) is caused by the reorganization of the initial powder packing at the beginning of the preshear process. After $40 \mathrm{~s}(1.3 \mathrm{~mm})$, a maximum density is reached, followed by some dilatancy (height increase of about 20 particle diameters) before the steadystate is reached after $100 \mathrm{~s}(2.6 \mathrm{~mm})$. The unloading (i.e. the shear drive is moved with the same rate in opposite direction, taking off in this way the force on the shear ring) allows the lid to compress the packing structure down to a even lower porosity (higher density) state. During the load change (up and down "jumps" in the figure), when the normal stress is reduced to the desired $\sigma_{n}$, the powder reacts by expanding to higher porosity (lower density). This response as function of the normal stress can be used to better understand the elastic properties of a consolidated (and presheared) powder continuum; however, this is far from the scope of this paper. 
At least qualitatively, one can see that the reproducibility of the results during pre-shear is very good, and that the "jumps" of the lid are higher for lower normal stress $\sigma_{n}$.

\section{SIMULATIONS AND RESULTS}

\subsection{Translational Shear Cell}

The translational (2D) shear cell studied by simulations in the following contains $N \approx 3500$

particles with radii randomly drawn from a homogeneous distribution with minimum $6 \mu \mathrm{m}$ and maximum $12 \mu \mathrm{m}$. The material properties correspond to those of titanium dioxide powder. The friction coefficient is $\mu=0.5$ and the shear rate is $v_{s}=2 \mathrm{~mm} / \mathrm{min}$. The simulations of the translational shear were performed with the linear adhesion contact law, as implemented in the commercial software (PFC2D, Itasca Inc.) already. With other words this law is the simplified version of the earlier discussed history dependent adhesion model (Luding 2001, 2003, Eq. (4), Fig. 10) for the case $k_{1}=k_{2}$. For convenience, a constant adhesion force $f_{0}$ between $1-10 \mathrm{mN}(0.1-1 \%$ of average contact forces during loading) was used to approximate the pull-off force (Tomas, 2001). Then a series of simulations is performed applying the more general dissipative contact model for adhesive particles (Eq. (4), Fig. 10) $\left(k_{1} \neq k_{2}\right)$, and the comparison is made.

\subsubsection{Shear zone}

In order to visualize the shear zone, vertical columns of particles were initially coloured likewise. Fig. 17 shows the system after shear both for the simulations and the experiment. For the latter, a cell built from two parts was used. With the help of concentric cylinders (transparencies) coloured and pure (white) $\mathrm{TiO}_{2}$ powder was filled in the cell and later, the powder bed was cut vertically through the center, after the shear test. The shear zone and the angle $\gamma$, the so-called shear distortion, are clearly recognised here. The angle $\gamma$ is defined as a function of the shear displacement $\mathrm{d} s$ and the shear zone height $\mathrm{d} h_{S Z}$. The simulation snapshot shows also the contact force network during the shearing with line thickness proportional to normal force. The force lines run mostly from the upper left wall, where the shear force acts, to the lower right wall, where the corresponding response force acts - the powder is thus strongly anisotropic. Examining the shear strain, more contacts and stronger forces are 
parallel to the compressive direction of shear (top-left to bottom-right). This correlates also with the fact, that the orientation of the major principal stress $\sigma_{l}$ is tilted counter-clockwise in the same direction - acting against the shear. The lower left and the upper right walls experience much weaker forces, correlated to the tensile strain direction (bottom-left to topright).
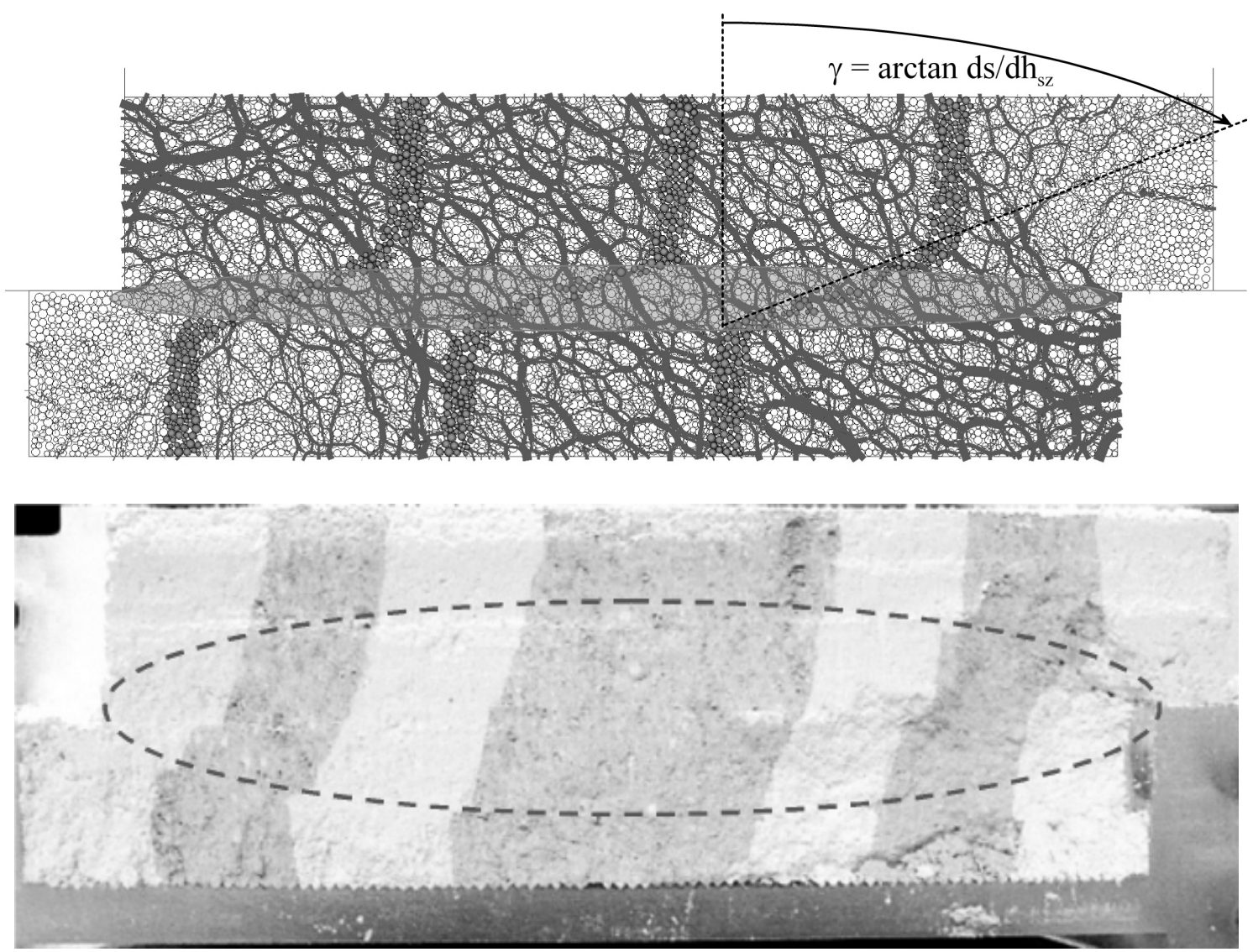

Fig. 17: Location of the shear zone and determination of shear distortion.

(Top) simulation, (Bottom) experiment

Fig. 18 shows the velocity vectors of every particle during later shearing with the line length proportional to the actual velocity. The motion takes place mostly in the upper ring, where the velocities are approximately equal. The steady-state flow with a stationary shear rate is reached at the end of the shear distortion. However, also short-time fluctuations and variations of the shear zone are evidenced. 


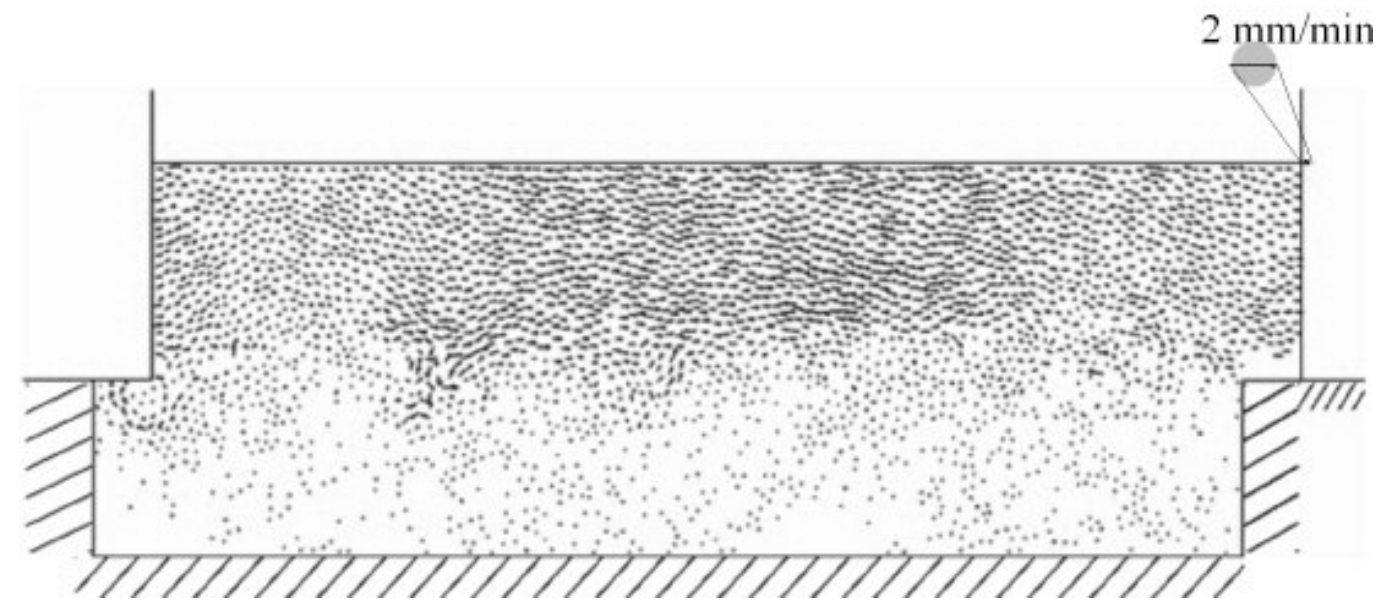

Fig. 18: Velocity vectors during the steady-state flow. The scale for the vectors is given top right.

\subsubsection{Shear test with varying initial porosity}

Fig. 19 shows the force-displacement diagram (left) and the volumetric strain (right) at a constant normal stress of $\sigma_{n}=3 \mathrm{kPa}$ for three different values of the porosity $\varepsilon=1-\rho_{b} / \rho_{s}$ (where $\rho_{b}$ is the bulk material density, and $\rho_{s}$ - the solid density), i.e., three preshear tests with different consolidation history. The volumetric strain $\varepsilon_{v}$ was defined in Eq. (14) and is given here in percent $(\%)$. The preparation of the different densities is explained in the following together with the discussion of the respective results.

The upper curves in both graphs are obtained at a two-dimensional porosity of the particle system of $\varepsilon_{2 D}=0.16$, which corresponds to about $\varepsilon_{3 D}=0.46$ for three dimensions (Deresiewicz 1958). The typical behaviour of "overconsolidated" powder is observed in this case, achieving the peak force after about $7 \mu \mathrm{m}$ shear, together with the first-stage compaction. For larger shear displacement, the shear force decreases and dilatancy is evidenced, before the steady state flow regime is reached later on, after a deformation of about $20 \mu \mathrm{m}$. The middle curves with $\varepsilon_{2 D}=0.18\left(\varepsilon_{3 D} \sim 0.50\right)$ show almost ideal steady-state flow behaviour, as also seen from the experiments, see Fig. 16. Note that the volumetric strain is coming to relatively stable state only at the end of the shear process, i.e., dilatancy stops later than for the overconsolidated sample, which is not in agreement with the theoretical expectations. However, the experiments with the Jenike shear cell show the same tendency (Fig. 16), even though dilatancy is much less pronounced. The fluctuations of the shear force can be explained by means of temporary and local shear-thickening and shear-thinning processes and the bad statistics obtained is due to the small numerical samples as compared to the real 
experiments. The lower curves correspond to $\varepsilon_{2 D}=0.20\left(\varepsilon_{3 D} \sim 0.54\right)$ and show the features of an "underconsolidated" powder: Both critical state stress and density are reached only after very large shear deformation, which shows that underconsolidated samples should not be used for short-shear devices like the Jenike cell.

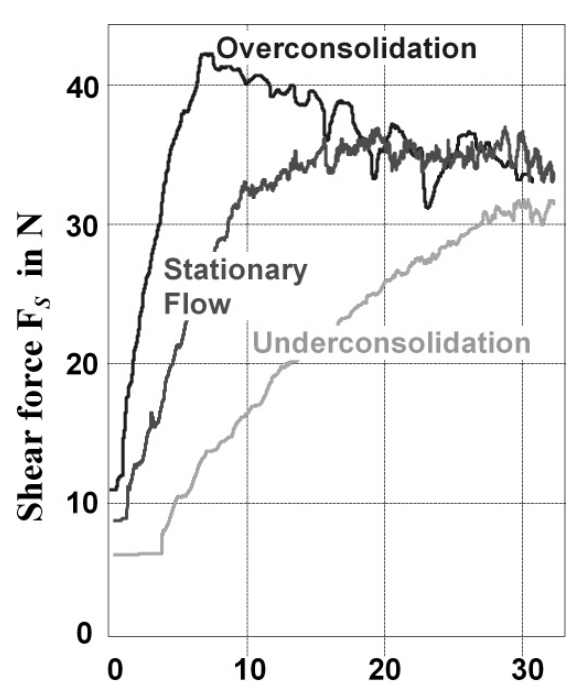

Shear displacement $\mathrm{s}$ in $\mu \mathrm{m}$

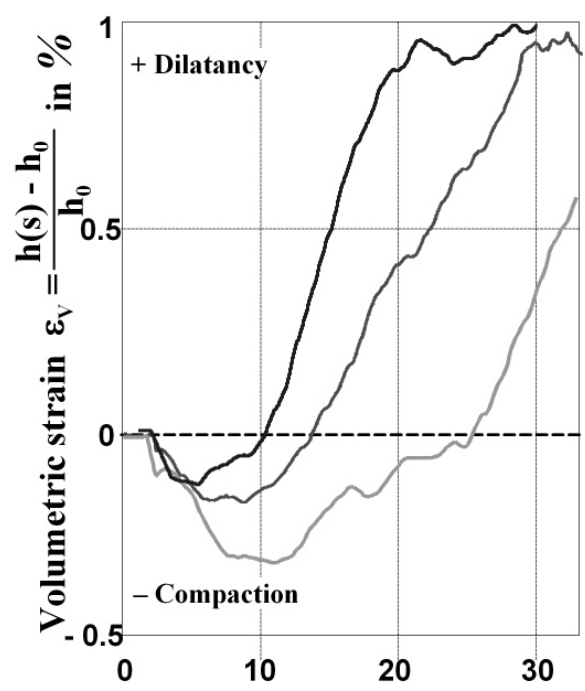

Shear displacement $\mathrm{s}$ in $\mu \mathrm{m}$

Fig. 19: (Left) Shear force-displacement diagram (steady-state flow) and (Right) Volumetric strain plotted against displacement for three different initial porosity values

Taking into account the shear testing experience above, good qualitative agreement is reached between the simulation results and the laboratory tests. As a final step, we have to adjust and compare the shear deformations and strains (measured in time for the experiments) and measured in $\mu \mathrm{m}$ for the simulations. The dimensionless shear strain from experiments $\varepsilon(t)=v_{s} t / d_{Z}$, where $d_{Z}=95 \mathrm{~mm}$ is the diameter of the shear cell, is $\varepsilon=(2 \mathrm{~mm} \mathrm{95s} / 60 \mathrm{~s} / 95 \mathrm{~mm})=$ $1 / 30$, after $95 \mathrm{~s}$, when the steady-state is clearly reached after a shear deformation of $3 \mathrm{~mm}$. The shear strain from simulations is $\varepsilon=(0.02 \mathrm{~mm} / 1 \mathrm{~mm})=1 / 50$, under similar conditions, when the steady state is reached after only $20 \mu \mathrm{m}$ shear displacement. Thus the shear strain required for steady state flow in the simulations is of the same order of magnitude as the one in the experimental results. Differences can be due to the two- and three-dimensional nature of simulations and experiments, respectively, due to the simplified contact models, and due to the much smaller size of the simulation system-volume and length: the number of particles simulated varied from 2000 up to 10000 , whereas for example titania powder in the used experimental shear cell, contains about $2 \times 10^{14}$ single particles. 


\subsubsection{Shear test with varying shear rate}

Concerning shear rates, the quasi-static regime was considered up-to now. A test whether a simulation is in fact performed in the slow, quasi-static regime, is to perform the simulation with a higher rate. If the simulations agree, the faster one can still be assumed quasi-static. This test was performed for the bi-axial box already by Luding (2001), and is performed here for the Jenike cell. Fig. 20 shows a stress-strain-diagram (top) and the relative volumetric strain (bottom) for three simulated shear tests with different shear rates $v_{s}$, starting from the critically consolidated initial configuration as discussed above. The simulations are carried out as the complete shear test procedure recommended by Jenike with the consolidation, preshear, unloading and shear steps. Here the normal stress is $\sigma_{\text {pre }}=3 \mathrm{kPa}$ for preshear and $\sigma_{n}=0.6 \mathrm{kPa}$ for shear. The two lower curves for shear rates of $v_{s}=0.03 \mathrm{~m} / \mathrm{s}$ and $v_{s}=0.3 \mathrm{~m} / \mathrm{s}$ are close to each other and show the typical behaviour of quasi-static, slow flowing powders (Tardos, 2003). In this case the shear stress does not depend on the shear rate and the Coulomb friction and cohesion influence the powder flow. The third curve $\left(v_{s}=3 \mathrm{~m} / \mathrm{s}\right)$ shows substantially higher shear stress and volumetric strain by the same normal stress (load) values. Therefore, the simulations with the highest shear rate could not be considered as the steadystate (dynamic equilibrium), while the moderate shear rate is close to quasi-static.

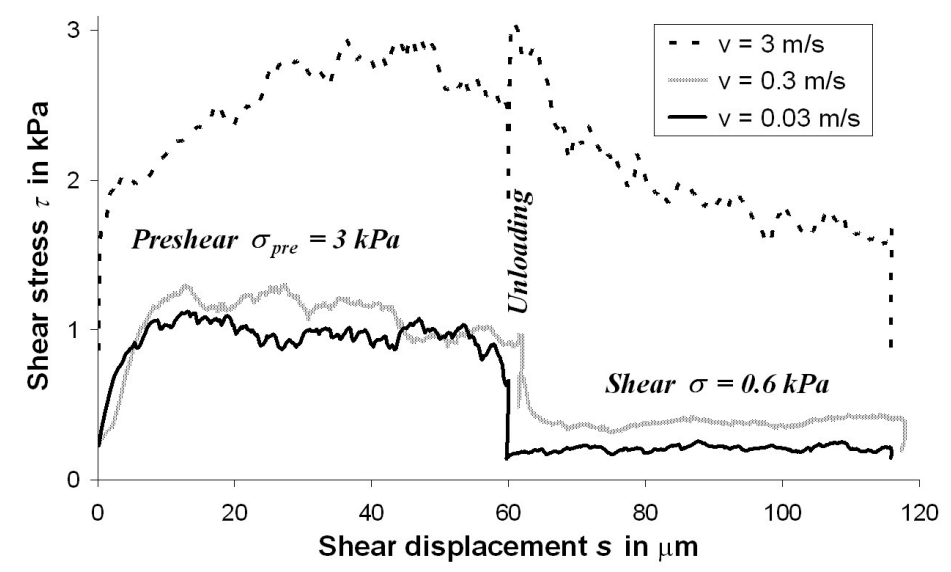




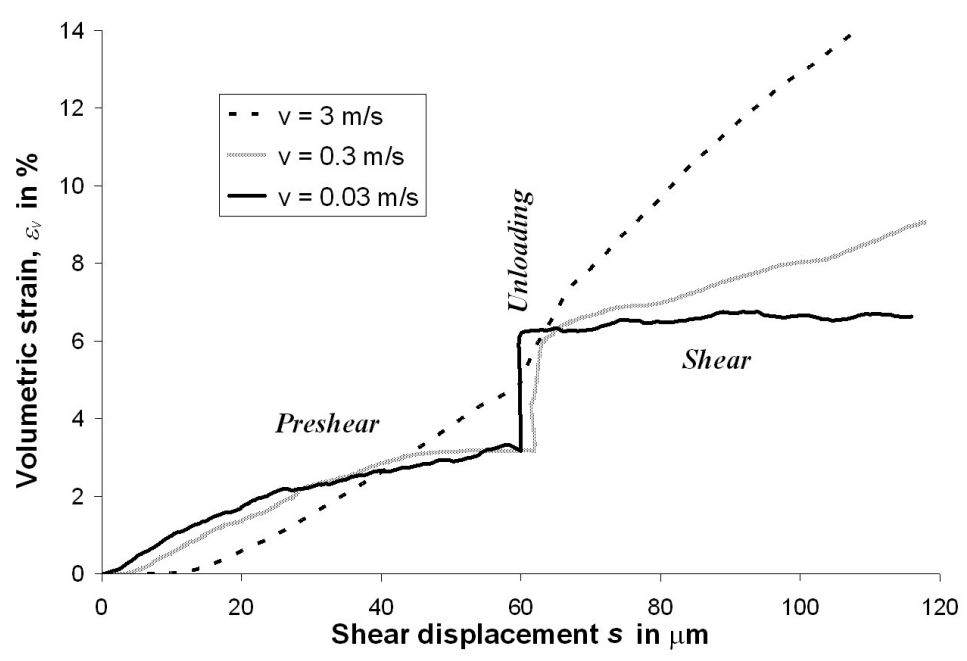

Fig. 20: Influence of the shear rate on the shear stress (Top) and volumetric strain (Bottom), both plotted against shear displacement.

\subsubsection{Shear test with varying particle adhesion - comparison with experiment}

From the steady-state shear stresses for different applied normal stresses, as obtained from the Jenike cell experiments and simulations, the yield locus can be defined. The slope of the yield locus gives the angle of internal friction of the material, and the intersection with the vertical axis gives the macroscopic cohesion in stress units. Fig. 21 shows the comparison of the yield locus calculated from the Jenike shear cell experiments with the simulated yield loci obtained applying different contact laws with somewhat different parameters. The experimental data (solid circles) show an internal friction angle of the powder "continuum" of $\varphi_{i}=26^{\circ}$, and a cohesion $\tau_{c}=3.09 \mathrm{kPa}$. The lowermost line is the result of the simulated shear test, where the simplest microscopic interaction between the particles was used, the linearspring-dashpot contact model in normal direction, with a constant adhesion force between the particles in contact $f_{0}=10 \mathrm{mN}$, see Fig. 10 . The preshear normal stress was $\sigma_{p r e}=20 \mathrm{kPa}$ and, for the shear process, the adhesion force values $f_{0}$ were taken proportional to the reduced macroscopic normal stresses. The average contact forces during preshear were in the range of 200-500 mN. The 2D data in Fig. 21 are obtained using the elastic-plastic contact model with variable adhesion (see Eq. (4), Fig. 10) with $k_{1}=10^{8} \mathrm{~N} / \mathrm{m}, k_{2}=2 k_{1}$, and the "microscopic adhesion stiffness" $k_{c}=0, k_{c}=k_{2}$, and $k_{c}=4 k_{2}$ (from bottom to top), the latter leading to the macroscopic result for the internal friction angle $\varphi_{i}=20^{\circ}$, and cohesion $\tau_{c}=1.13 \mathrm{kPa}$. Thus, 
comparison of the simulated 2D data with experiments does not yet yield satisfactory quantitative agreement for this parameter set.

That is why a series of simulations with input data of the last 2D simulation was performed in three dimensions (dashed line), leading to nearly perfect quantitative agreement with the experiments. The same tendency of increase of macroscopic cohesion with the contact model adhesion was observed also in further 3D simulations (data not shown). Therefore, the implemented hysteretic contact model with variable adhesion is closer to reality for the studied fine powders in order to mimic the macroscopic flow behaviour of a consolidated powder bed, when compared to the oversimplified linear contact model with load independent adhesion.

Together with other results (Luding 2001, Luding 2003, Luding 2004b, Luding 2004c, Luding 2005) on the fact that the macroscopic cohesion $\tau_{c}$ can be related to the maximal microscopic attractive force $f_{\min }$, one can foresee that the 2D simulations should use about three times larger typical adhesion forces. Note that (due to the contact model) this has to be achieved by using smaller ratios $k_{1} / k_{2}$ - increasing $k_{c}$ further does not lead to considerably stronger adhesion for the parameters used above. 


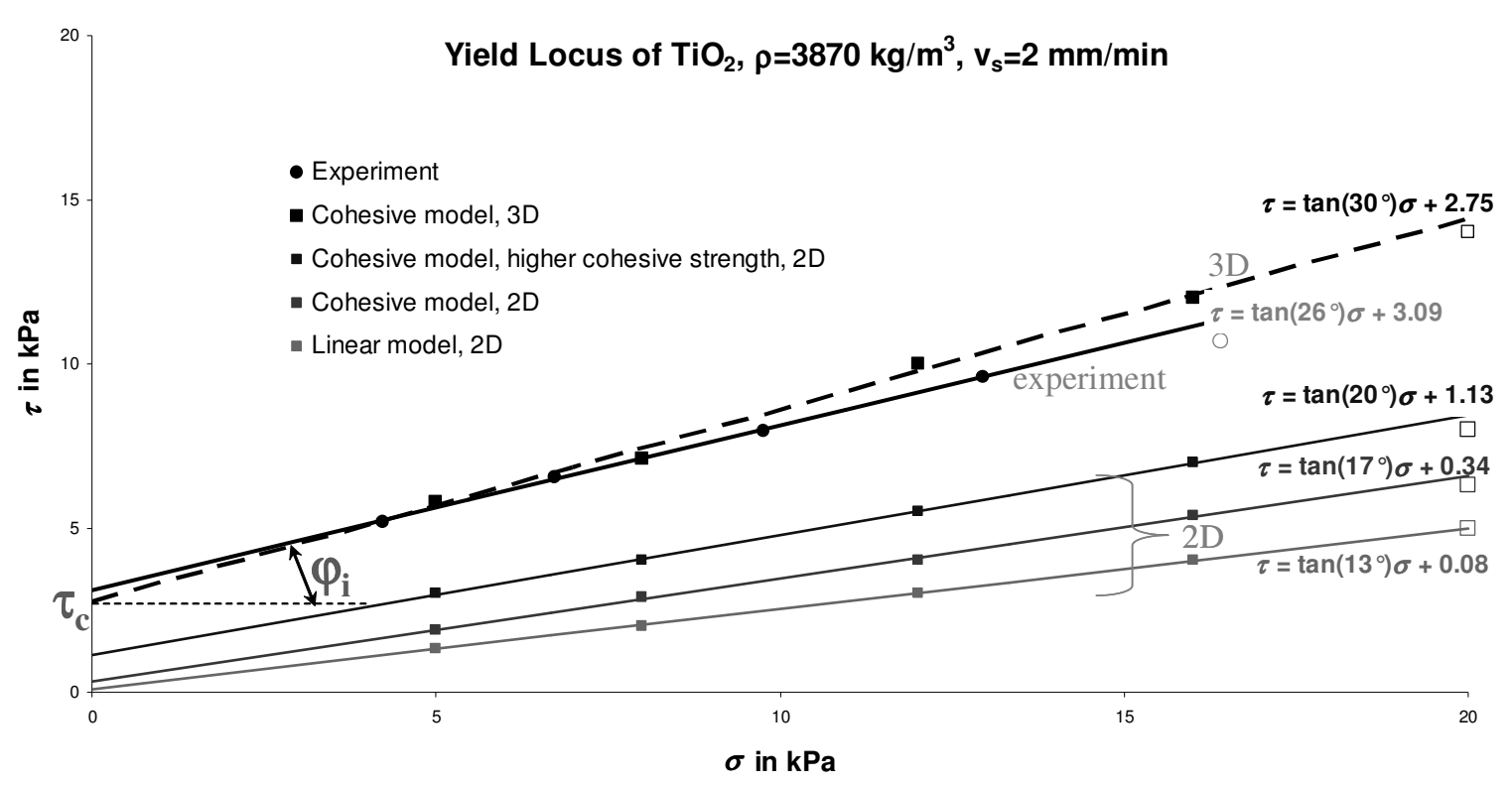

Fig. 21: Yield locus in a shear stress plotted against normal stress graph for the experiments (material $\mathrm{TiO}_{2}$ ) and different microscopic force-displacement laws (linear and history dependent adhesion) used in simulations

\subsection{Biaxial box}

The bi-axial box system examined in the following contains $N=1950$ particles with radii randomly drawn from a homogeneous distribution with minimum $0.5 \mathrm{~mm}$ and maximum 1.5 $\mathrm{mm}$. The friction coefficient used in the two-dimensional simulations is $\mu=0.5$, if not explicitly specified. The total mass of the particles in the system is about $0.02 \mathrm{~kg}$, shear rate (compression velocity) $v_{z}=0.6 \mathrm{~mm} / \mathrm{min}$. In the biaxial box simulations, the jump-in force in the particle contact constitutive model (see Fig. 10) is here $f_{0}=0$. If not explicitly mentioned, the material stiffness parameters are $k_{2}=10^{5} \mathrm{~N} / \mathrm{m}, k_{1} / k_{2}=1 / 2, k_{\mathrm{c}} / k_{2}=0$, and the contact-viscosity is $0.1 \mathrm{~kg} / \mathrm{s}$. The eigen-frequency of the particle contact is hence typically $10^{-5} \mathrm{~s}$ so that an integration time-step of $2.10^{-7} \mathrm{~s}$ is used, in order to allow for a "safe" integration.

\subsubsection{Shear zone}

Fig. 22 shows the force network during shear, (Left) at the maximum value of vertical stress $\sigma_{z z}$, which is approximately 2.5 times higher than the corresponding horizontal stress $\sigma_{x x}$; and (Right) after fully forming the shear zone, i.e., in steady state flow. During compression, the 
initially isotropic material becomes more and more anisotropic (Luding, 2005), i.e., more and stronger contacts form in vertical direction - acting against the compression. After the shear zone is formed the homogeneous state of stresses is broken, showing now strong inhomogeneity: some of the force chains are much stronger or weaker inside/along the shear band than in the bulk.

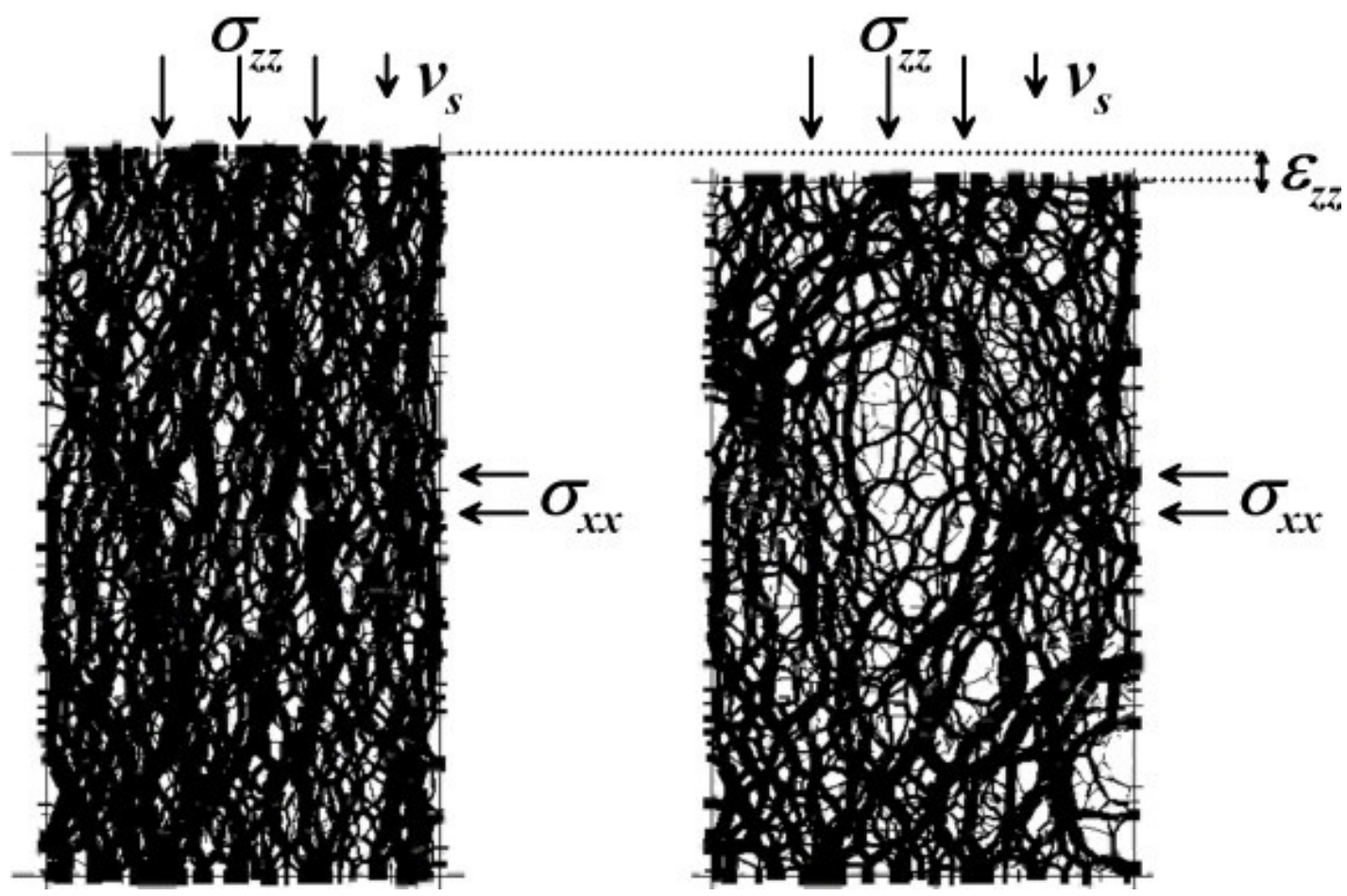

Fig. 22: Contact force distribution (each line represents a contact and the line thickness is proportional to the absolute value of the force at this contact) when (Left) the maximum vertical stress is reached, and (Right) the shear zone is formed and fully developed.

Another possibility to determine the shear zone (extent and direction) is to have a look at either the accumulated particle rotations (Fig. 23 left) at the end of the shearing or the current particle displacement vectors (Fig. 23 right). This shows that rotations are only active in the shear zone and that the shear zone is localized from top-left to bottom-right. The shear band is here much narrower than compared to the wide shear band obtained in the translational shear cell simulations and in Jenike experiments. 


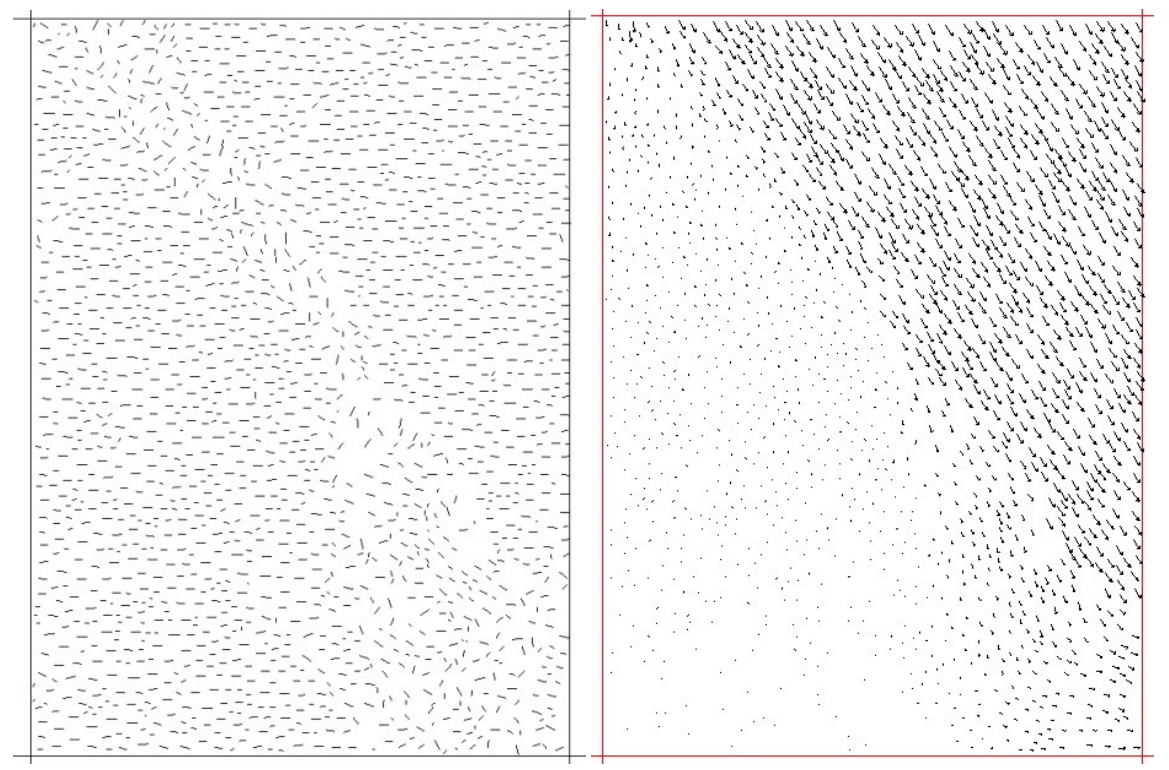

Fig. 23: Location of the shear zone: (Left) Accumulated particle rotations (horizontal lines show that rotation did not take place outside the shear zone), (Right) particle velocity vectors

\subsubsection{Biaxial test without adhesion, with friction and varying pressure}

In Fig. 24 (Left), the volume change of a typical simulation shows first compression, then dilatancy, and eventually almost no change at very large deformations, up to 15 percent. At the same time, the stress response, in Fig. 24 (Right) (where the indices $\mathrm{xx}$ and $\mathrm{zz}$ denote horizontal and vertical stresses, respectively), shows elastic, softening, and critical state flow behaviour. For both volumetric strain and stresses, large fluctuations are evidenced in the critical state, while the data are relatively smooth before. 

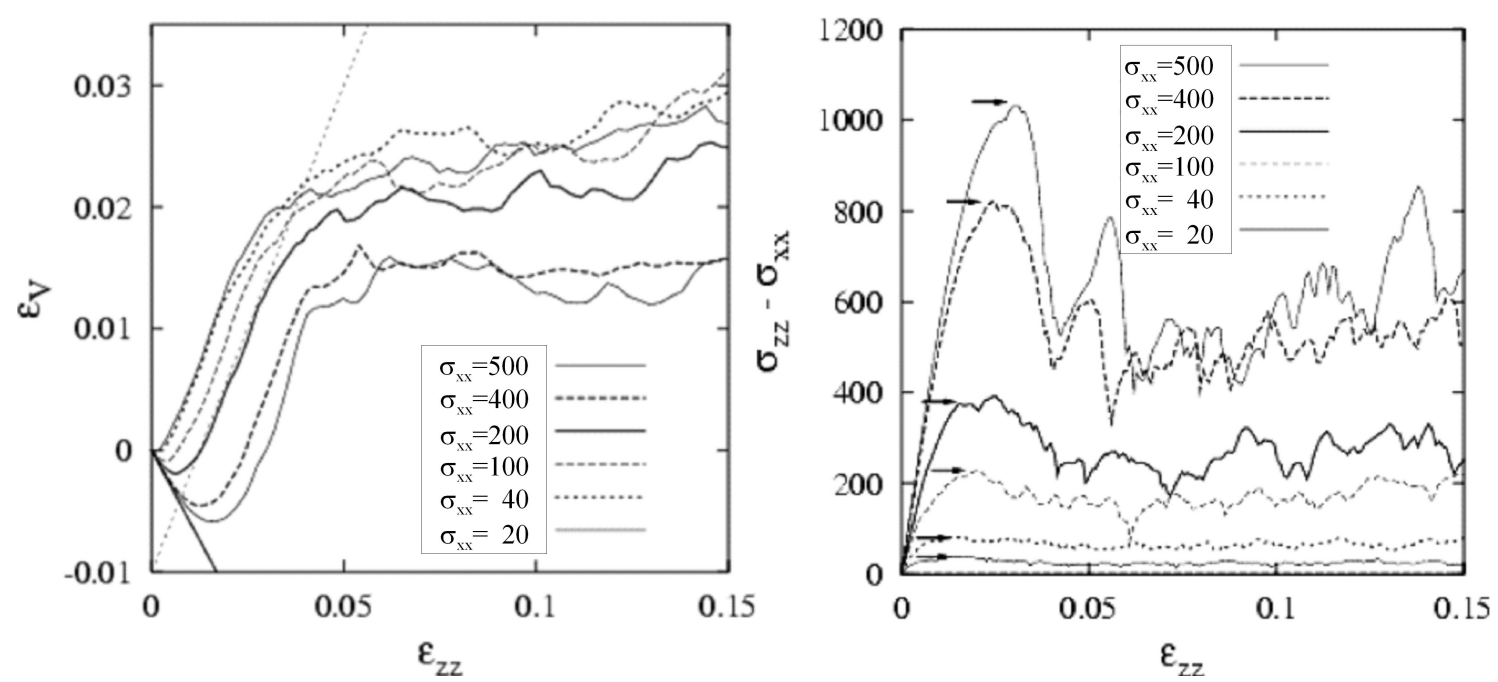

Fig. 24: Volumetric strain (Left) and stress-difference (Right) during large deformations, both plotted against vertical strain, for different side pressures as indicated in the inset. The peak yield stress is marked by arrows.

First, the stress-difference increases linearly; then the slope gradually decreases (softening), until the stress reaches its maximum (peak yield stress). After the peak, further softening/weakening behaviour (with negative slope) is followed by an almost constant, but strongly fluctuating stress for larger deformations.

The flow behaviour of the system can be quantified by plotting Mohr-circles for the maximum vertical stress (right-most point on the circle) for different confining pressures (leftmost point), see Fig. 25. The stress-difference then corresponds to the diameter of the circle. In contrast to the Jenike cell geometry - where nothing can be said about - the eigendirections of the system are parallel to the walls in a bi-axial box with no friction active between particles and walls. The left- and right-most points on the circles are then indeed corresponding to the wall stresses and no shear stress is active, rendering the stress tensor diagonal. The tangent to the circles (slope 0.588) can be seen as the flow function for peak stress. The corresponding friction angle (inverse tan) is about $30.5^{\circ}\left(26.6^{\circ}\right.$ expected from micro-friction). It is linear for the examined parameters, with its slope only slightly larger than expected from the microscopic friction at the contacts alone. Since we have not used adhesion forces here, the macroscopic the flow function hits the origin, i.e., $\tau_{c}=0$. 


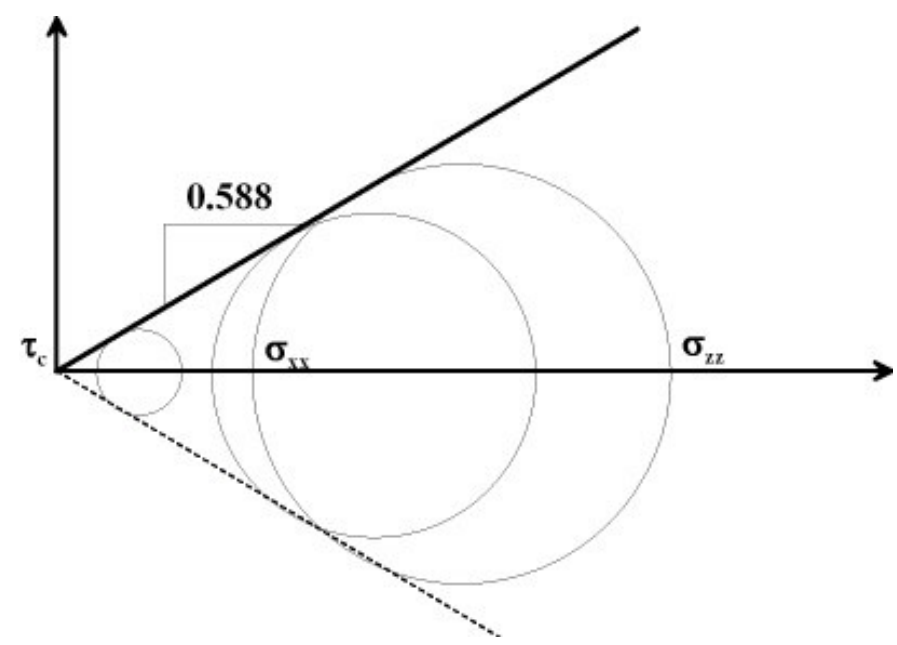

Fig. 25: Mohr circle representation of the yield locus at peak stress, see the arrows in Fig. 24 (right).

In Fig. 26 (Left), the volume change from simulations with different friction coefficients shows first compression (magnitude increasing with $\mu$ ), then dilatancy (slope increasing with $\mu$ ), and eventually saturation (strain level increasing with $\mu$ ) with a very weak change at large deformations. The stress response, shown in Fig. 26 (Right), shows again elastic, softening, and critical state flow behaviour. With increasing friction, the peak stress and the softening magnitude increase systematically. The critical state stress is increasing less strong as a function of $\mu$.
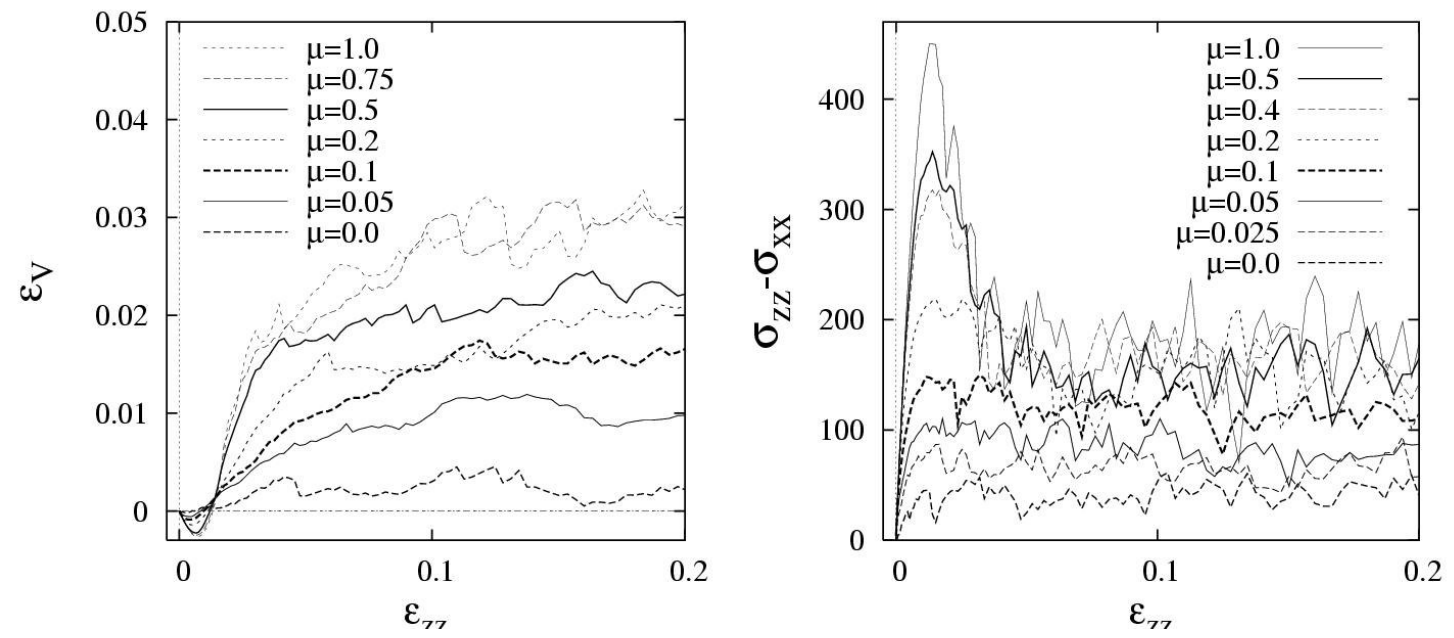

Fig. 26: Volumetric strain (Left) and stress-difference (Right) during large deformations, both plotted against the vertical strain, for different friction coefficients as indicated in the inset, with side stress $=\sigma_{\mathrm{xx}}=200$. All these samples were prepared without friction - leading to 
over-consolidation, i.e., rather high densities - and friction was set to the desired value only at the beginning of the bi-axial shear deformation.

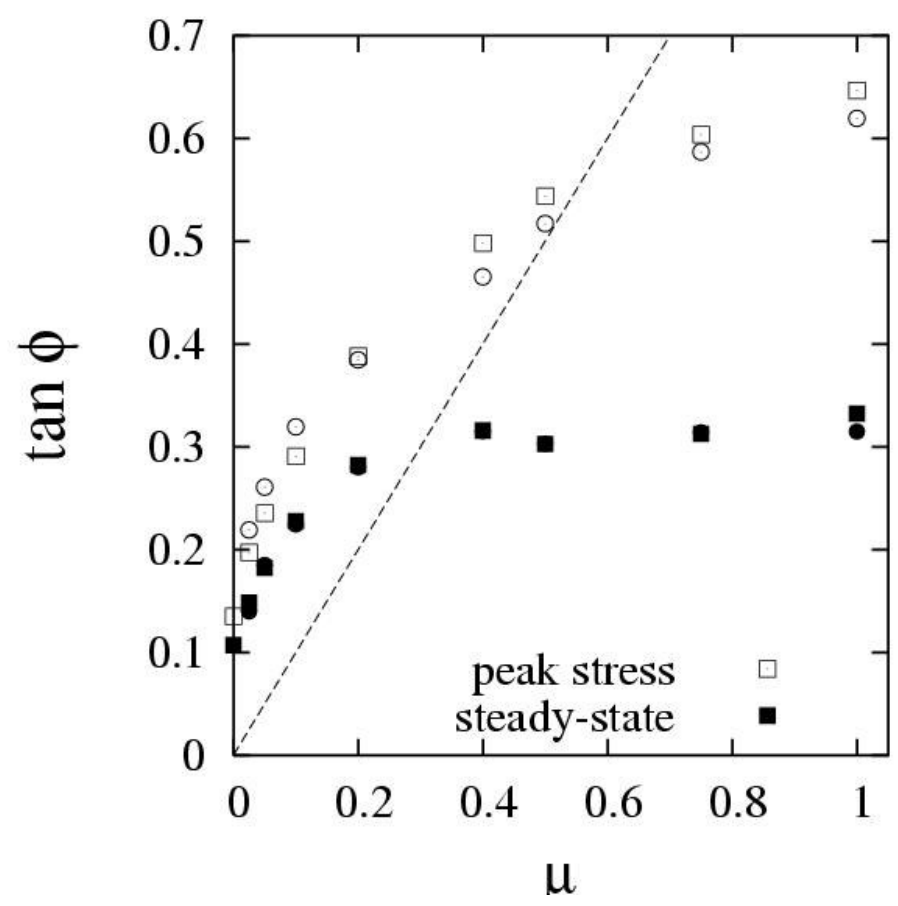

Fig. 27: Macroscopic friction coefficient, as obtained from the biaxial box simulations, plotted as function of the microscopic contact friction coefficient. The preparation of the samples was done with $\mu=0$ (squares) or with $\mu=0.5$ (circles). The solid symbols give the effective friction angle in the steady state flow regime.

In Fig. 27, the macroscopic friction is plotted against the microscopic friction coefficients, for different preparation procedures, either using $\mu_{\text {prep }}=0$ or $\mu_{\text {prep }}=0.5$. The density obtained for preparation with $\mu_{\text {prep }}=0(0.8467)$ is a little larger than the density of a packing prepared with finite friction (0.8445). In both cases, the friction was set to the desired value of $\mu$ (see inset) at the beginning of the deformation (shear) test. In the latter case, for the smaller friction values, this leads to a restructuring (densification to volume fractions up to 0.8461 for $\mu=0.025$ ) because some of the sticking contacts can slide when friction is reduced that abruptly. The steady state friction saturates at considerably lower values and does not depend on the preparation procedure. 


\section{SUMMARY AND CONCLUSIONS}

In summary, a set of DEM simulations based on experimentally inspired contact models was presented, and several macroscopic material parameters like, e.g., cohesion and friction angle, were extracted from the simulations. The experimental set-up, concerning the microscopic measurements of particle interactions based on AFM, was presented as well as details about macroscopic shear tests of powders.

Altogether this is a first step of a micro-modelling approach for cohesive frictional powders by means of going the whole long way from:

(i) measuring the interaction between ultra-fine particles,

(ii) implementing these complex "microscopic" contact constitutive laws,

(iii) modelling the shear dynamics of many-particle systems, and

(iv) finding out the macroscopic flow rules and parameters of powders.

While in earlier studies, the macroscopic cohesion could be related to the maximal microscopic attractive force that is accessible experimentally (Luding 2001, 2003), the macroscopic friction of the powder is also related to the contact-friction, but in a much more subtle, non-linear way: The powder continuum shows macroscopic friction even without micro-friction (due to geometric interlocking and subsequent shear-resistance of the powder particles) and the micro friction does not affect the macroscopically determined friction above some value, because in the case of extremely strong micro-friction, the particles just prefer to roll and thus can shear.

Microscopic insights as observed from the discrete element simulations (like the peculiar force/stress-patterns) were helpful to identify the main control parameters for the further calculations. Also the predicted particle redistribution in the shear zone was verified by the experiments. Even though the simulated yield loci of the model powder (with back calculated parameters) in the Jenike shear cell simulations did not lead to satisfactory quantitative agreement with the measurements, so far, good qualitative agreement of the simulations with reference experiments is reached in 2D. Quantitative agreement with physically based (back calculated) contact model parameters could be reached in 3D. Possible reasons for the quantitative disagreement are the difference in dimension (2D in simulations vs. 3D in experiments), the idealized nature of the particles in the simulations, and details of the simplified contact model used. Non-spherical particles can be formed as unbreakable clumps (agglomerates) of spherical primary ones (Khanal 2004). Surface roughness can be modelled 
by "rolling friction", as implemented and tested already (Luding 2007). In conclusion, the history dependent adhesion contact model shows a "positive" influence on the macroscopic flow behaviour of a consolidated powder bed. It seems to be capable of simulating the cohesive properties of a material with variable adhesion (pull-off) force depending on the preconsolidation history on every inelastic particle contact.

Further material parameters have to be identified, and also the role of particle rotations is an open issue, as related to advanced micro-polar constitutive models (Tejchmann 1993). In both simulation and experiment, rotations are active in the shear band where the rotational degree of freedom is activated. The corresponding parameter identification and the micro-macrotransition for anisotropic micro-polar continuum models is a challenge for the future, like the implementation and simulation of experimentally determined force-laws in three-dimensional systems.

\section{ACKNOWLEDGEMENT}

The authors acknowledge the German Research Foundation (DFG) for the financial support of the research group "Behaviour of Granular Media".

\section{REFERENCES}

Allen, M. P., Tildesley, D. J., (1989). Computer Simulation of Liquids. Oxford University Press.

Antonyuk, S., Tomas, J., Heinrich, S., Mörl, L., (2005). Breakage behaviour of spherical granulates by compression, Chem. Eng. Sci. 60, 4031-4044.

Bartels, G., Unger, T., Kadau, D., Wolf, D. E., Kertesz, J., (2005), The effect of contact torques on porosity of cohesive powders. Granular Matter 7, 139.

Binnig, G., Quate, C. F., Gerber, C., (1986). Atomic force microscope. Phys. Rev. Lett. 56, 930 - 933.

Black, W., de Jongh, J.G.V., Overbeek, J.T.G., Sparnaay, M.J., (1960). Measurements of retarded Van Der Waals' forces. Trans. Faraday Soc. 56, 1597-1608. 
Böhme, G., Krupp, H., Rabenhorst, H., Sandstede, G., (1962). Adhesion measurements involving small particles. Trans. Inst. Chem. Engrs. (London) 40, 252.

Bonaccurso, E., Kappl, M., Butt, H.-J., (2005). Hydrodynamic force measurements: Boundary slip of water on hydrophilic surfaces and electrokinetic effects. Phys. Rev. Lett. (in press).

Bowen, R. W., Doneva, T. A., (2000). Atomic force microscopy studies of membranes. Effect of surface roughness on double-layer interactions and particle adhesion. J. Colloid Interface Sci. 229, 544 - 549.

Bradley, R.S., (1932). The cohesive force between solid surfaces and the surface energy of solids. Phil. Mag. 13, 853-862.

Braithwaite, G. J. C., Luckham, P. F., (1997). Effect of molecular weight on the interactions between poly(ethylene oxide) layers adsorbed to glass surfaces. J. Chem. Soc.-Faraday Trans. 93, 1409 - 1415.

Butt, H.-J., (1991). Measuring electrostatic, van der Waals, and hydration forces in electrolyte solutions with an atomic force microscope. Biophys. J. 60, 1438-1444.

Butt, H.-J., (1994). A technique to measure the force between a colloidal particle in water and a bubble. J. Colloid Interface Sci. 166, 109 - 117.

Butt, H.-J., Jaschke, M., Ducker, W.A., (1995). Measuring surface forces in aqueous solution with the atomic force microscope. Bioelectrochem. Bioenerg. 381, 91 - 201.

Cain, R. G., Page, N. W., Biggs, S., (2000). Microscopic and macroscopic effects of surface lubricant films in granular shear. Phys. Rev. E 62, 8369 - 8379.

Capella, B., Dietler, G., (1999). Force-distance curves by atomic force microscopy. Surf. Sci. Reports 34, 1 - 104.

Castellanos, A., (2005). The relationship between attractive interparticle forces and bulk behavior in dry and uncharged fine powders. Advances in Physics 54(4), 263-376.

Cho, J. M., Sigmund, W.M., (2002). Direct surface force measurement in water using a nanosize colloidal probe technique. J. Colloid Interface Sci. 245, 405 - 407.

Craig, V. S. J., Hyde, A. M., Pashley, R. M., (1996). Application of the light-lever technique to the study of colloidal forces. Langmuir 12, 3557 - 3562.

Cundall, P.A., Strack, O. D. L., (1979). A discrete numerical model for granular assemblies. In Geotechnique, 29, 47-65.

D'Addetta, G. A., Kun, F., Ramm, E., (2002). On the application of a discrete model to the fracture process of cohesive granular materials, Granular Matter 4 (2), 77-90.

Deresiewicz, H., (1958). Mechanics of granular matter, Advances in Appl. Mech. 5, 233-306. 
Derjaguin, B.V., Abrikosova, I.I., Lifshitz, E.M., (1956). Direct measurement of molecular attraction between solids separated by a narrow gap. Quart. Rev. Chem. Soc. 10, 295-329.

Derjaguin, B. V., Muller, V. M., Toporov, V. J., (1975). Effect of contact deformations on the adhesion of particles, Colloid Surf. A-Physicochem. Eng. Asp. 53, 314 - 326.

Ducker, W.A., Senden, T. J., Pashley, R. M., (1991). Direct measurement of colloidal forces using an atomic force microscope. Nature 353, 239-241.

Ducker, W.A., Senden, T. J., Pashley, R. M., (1992). Measurement of forces in liquid using a force microscope. Langmuir 8, 1831-1836.

Ecke, S., Butt, H.-J., (2001). Friction between individual microcontacts. J. Colloid Interface Sci. $244,432-435$.

Ecke, S., Raiteri, R., Bonaccurso, E., Reiner, C., Deiseroth, H. J., Butt, H. J., (2001).

Measuring normal and friction forces acting on individual fine particles. Rev. Sci. Instrum. $72,4164-4170$.

Feiler, A., Larson, I., Jenkins, P., Attard, P., (2000). A quantitative study of interaction forces and friction in aqueous colloidal systems, Langmuir, 16, 10269-10277.

Heim, L. O., Blum, J., Preuss, M., Butt, H. J., (1999). Adhesion and friction forces between spherical micrometer-sized particles. Phys. Rev. Lett. 83, 3328 - 3331.

Hertz, H., (1882). Über Die Berührung Fester Elastischer Körper. J. reine u. angew. Math. 92, 156-171.

Hüttl, G., Beyer, D., Müller, E., (1997). Investigation of electrical double layers on SiO2 surfaces by means of force vs. distance measurements. Surf. Interface Anal. 25, 543-547.

Israelachvili, J.N., Tabor, D., (1972). The Measurement of Van Der Waals Dispersion Forces in the Range 1.5 to $130 \mathrm{~nm}$. Proc. R. Soc. Lond. A 331, 19-38.

Israelachvili, J.N., Tabor, D., (1973). Progress in Surface and Membrane Science, edited by J. F. Danielli, M. D. Rosenberg, and D. A. Cadenhead (Academic Press, New York, 1973), 7, $1-55$.

Israelachvili, J.N., Adams, G.E., (1978). Measurements of forces between two mica surfaces in aqueous electrolyte solutions in the range 0-100 nm. J. Chem. Soc. Faraday Trans. I 74, 975-1001.

Israelachvili, J.N., (1992). Intermolecular and Surface Forces, Academic Press, London. Jenike, A.W., (1964). Storage and flow of solids, Eng. Exp. Station, Bull. 123, Univ. Utah.

Johnson, K. L., Kendall, K., Roberts, A. D., (1971). Surface energy and the contact of elastic solids. Proc. R. Soc. London, Ser. A 324, 301 - 313. 
Kappl, M., Butt, H.-J., (2002). The Colloidal Probe Technique and its Application to Adhesion Force Measurements, Part. Part. Syst. Charact. 19, 129-143.

Khanal, M., Raj, R., Schubert, W., Tomas, J., (2004). DEM simulation of clumps shapes on cracking, Vortrag, GVC-Fachausschuß “Zerkleinern”, Braunschweig.

Kordecki, M. C., Orr, C., (1960). Arch. Env. Health 1, 1.

Kruyt, N. P., Rothenburg, L., (2001). Statistics of the elastic behaviour of granular materials. Int. J. of Solids and Structures 38, 4879-4899.

Larsen, R. I., (1958). The adhesion and removal of particles attached to air filter surfaces. Am. Indust. Hyg. J. 19, 265-270.

Luding, S., (1998). Collisions \& contacts between two particles. In H. J. Herrmann, J.-P. Hovi, and S. Luding, editors, Physics of dry granular media - NATO ASI Series E350, 285, Dordrecht, 1998. Kluwer Academic Publishers.

Luding, S., Lätzel, M., Volk, W., Diebels, S., Herrmann, H.J., (2001). From discrete element simulations to a continuum model, Comp. Meth. Appl. Mech. Engng. 191, 21-28.

Luding, S., Tykhoniuk, R., Tomas, J., (2003). Anisotropic material behaviour in dense, cohesive powders, CET (Chemical Engineering Technology) 26 (12), 1229-1232.

Luding, S., (2003). The micro-macro mechanics of granular materials. GACM report 2, 22 28.

Luding, S., (2004a). Molecular Dynamics Simulations of Granular Materials. In: The Physics of Granular Media, H. Hinrichsen and D. Wolf, eds., Wiley-VCH, Weinheim, 299-324.

Luding, S., (2004b). Micro-macro transition for an-isotropic, frictional granular packings. Int. J. Sol. Struct. 41(21), 5821-5836.

Luding, S., (2004c) Micro-Macro Models for Anisotropic Granular Media. In: Modelling of Cohesive-Frictional Materials, P. A. Vermeer, W. Ehlers, H. J. Herrmann, E. Ramm (eds.), A. A. Balkema, Leiden, 2004, 195-206.

Luding, S., (2005). Anisotropy in cohesive, frictional granular media. J. Phys.: Condens. Matter 17, 2623-2640.

Luding, S., (2007). Cohesive frictional powders: Contact models for tension. submitted to Granular Matter

Manual of PFC2D, ITASCA, http://www.itascacg.com/pfc.html

Meyer, E., Heinzelmann, H., Grötter, P., Jung, T., Hidber, H. R., Rudin, H., Güntherodt, H. J., (1989). Atomic force microscopy for the study of tribology and adhesion. Thin Solid Films $181,527-544$. 
Mindlin, R.D., Deresiewicz, H., (1953). Elastic spheres in contact. under varying oblique forces. Trans. ASME J. Appl. Mech. 20, 327-344.

Newton, J. M., Lam, K. K., (1992a). Effect of temperature on particulate solid adhesion to a substrate surface. Powder Technol. 73, 267 - 274.

Newton, J. M., Lam, K. K., (1992b). Influence of particle size on the adhesion behaviour of powders, after application of an initial press-on force. Powder Technol. 73, 117 - 125.

Newton, J. M., Lam, K. K., (1993). The influence of the time of application of contact pressure on particle adhesion to a substrate surface. Powder Technol. 76, 149 - 154.

Oda, M., Iwashita, K., (2000). Study on couple stress and shear band development in granular media based on numerical simulation analyses, Int. J. of Enginering Science 38, 17131740 .

Pierce, M., Stuart, J., Pungor, A., Dryden, P., Hlady, V., (1994). Adhesion force measurements using an atomic force microscope upgraded with a linear position sensitive detector. Langmuir 10, 3217 - 3221.

Podczeck, F., Newton, J. M., James, M. B., (1994). Assessment of adhesion and autoadhesion forces between particles and surfaces. 1. The investigation of autoadhesion phenomena of salmeterol xinafoate and lactose monohydrate particles using compacted powder surfaces. J. Adhes. Sci. Technol. 8, 1459 - 1472.

Podczeck, F., Newton, J. M., James, M. B., (1995a). Adhesion and autoadhesion measurements of micronized particles of pharmaceutical powders to compacted powder surfaces. Chem. Phar. Bull. 43, 1953 - 1957.

Podczeck, F., Newton, J. M., James, M. B., (1995b). Adhesion and friction between powdersand polymer or aluminium surfaces determined by a centrifuge technique. Powder Technol. 83, $201-209$.

Podczeck, F., Newton, J. M., James, M. B., (1995c). Assessment of adhesion and autoadhesion forces between particles and surfaces. 1. The investigation of adhesion phenomena of salmeterol-xinafoate and lactose monohydrate particles in particle-onparticle and particle-on-surface contact. J. Adhes. Sci. Technol. 9, 475 - 486.

Podczeck, F., Newton, J. M., (1995). Development of an ultracentrifuge technique to determine the adhesion and friction properties between particle sand surfaces. J. Pharmaceutical Sci. 84, 1067 - 1071. 
Podczeck, F., (1997). The development of a cascade impactor simulator based on adhesion force measurements to aid the development of dry powder inhalations. Chem. Pharm. Bull. 45, 911 - 917.

Podczeck, F., Newton, J. M., James, M. B., (1997). Influence of relative humidity of storage air on the adhesion and autoadhesion of micronized particles to particulate and compacted powder surfaces. J. Colloid Interface Sci. 187, 484 - 491.

Podczeck, F., (1999). Investigations into the reduction of powder adhesion to stainless steel surfaces by surface modification to aid capsule filling. Int. J. Pharm. 178, 93 - 100.

Polke, R., (1968). Haftung von Feststoffteilchen an Festkörperoberflächen bei milden Sinterbedingungen. Chem. Ing. Techn. 40, 1057-1060.

Polke, R., (1969). Adhesion of solids at elevated temperatures. Adhesion et Physico-Chimie des Surfaces Solides, 51-54.

Preuss, M., Butt, H.-J., (1998). Direct measurement of particle-bubble interactions in aqueous electrolyte: Dependence on surfactant. Langmuir 14, 3164 - 3174.

Raiteri, R., Preuss, M., Grattarola, M., Butt, H.-J., (1998). Preliminary results on the electrostatic double-layer force between two surfaces with high surface potentials. Colloid Surf. APhysicochem. Eng. Asp. 136, 191 - 197.

Rennie, P. R., Chen, X. D., Mackereth, A. R., (1998). Adhesion characteristics of whole milk powder to a stainless steel surface. Powder Technol. 97, 191 - 199.

Rouweler, G.C.J., Overbeek, J.T.G., (1971). Dispersion forces between fused silica objects at distances between 25 and $350 \mathrm{~nm}$. Trans. Faraday Soc. 67, 2117-2121.

Savkoor, A. R., and Briggs, G. A. D., (1977). The effect of a tangential force on the contact of elastic solids-in adhesion. Proc. Roy. Soc. Lond. A 356 103-114

Schaefer, D. M., Carpenter, M., Gady, B., Reifenberger, R., Demejo, L. P., Rimai, D. S., (1995). Surface roughness and its influence on particle adhesion using atomic force techniques. J. Adhes. Sci. Technol. 9, 1049 - 1062.

Schaefer, D. M., Carpenter, M., Reifenberger, R., Demejo, L. P., Rimai, D. S., (1994). Surface force interactions between micrometersize polystyrene spheres and silicon substrates using atomic force techniques. J. Adhes. Sci. Technol. 8, 197 - 210.

Tardos, G. I., McNamara, S., Talu, I., (2003). Slow and intermediate flow of a frictional bulk powder in the Couette geometry, Powder Technology 131, 23-39.

Tejchman, J., Wu, W., (1993). Numerical study on patterning of shear bands in a Cosserat continuum, Acta Mechanica 99: 61-74. 
Thornton, C., Antony, S. J., (2000). Quasi-static deformation of a soft particle system, Powder Technology 109 (1-3), 179 - 191.

Toikka, G., Spinks, G. M., Brown, H. R., (2001). Fine particle adhesion measured at elevated temperatures using a dedicated force rig. Langmuir 17, 6207 - 6212.

Tomas, J., (1999). Proc. Intern. Symp. on Reliable Flow of Particulate Solids III, 641-656, Porsgrunn.

Tomas, J., (2000). Particle Adhesion Fundamentals and Bulk Powder Consolidation. KONA - Powder and Particle 18, 157-169.

Tomas, J., (2001). Assessment of mechanical properties of cohesive particulate solids - part 1: particle contact constitutive model, Particulate Science \& Technology 19, 2, 95-110.

Tomas, J., (2002). Zur Mechanik trockener kohäsiver Schüttgüter, Schüttgut 8, 6, 522-537.

Tomas, J., (2003). Mechanics of nanoparticle adhesion - a continuum approach, in K.L. Mittal (Ed.), Particles on Surfaces 8: Detection, Adhesion and Removal, VSP Utrecht, 183229.

Tykhoniuk, R., Luding, S., Tomas, J., (2004). Simulation der Scherdynamik Kohäsiver Pulver, Chemie Ingenieur Technik, 76, 1-2, 59-62.

Weisenhorn, A. L., Hansma, P. K., Albrecht, T. R., Quate, C. F., (1989). Forces in atomic force microscopy in air and water, Appl. Phys. Lett., 54, 2651 - 2653.

Zauscher, S., Klingenberg, D. J., (2001). Friction between cellulose surfaces measured with colloidal probe microscopy. Colloid Surf. A-Physicochem. Eng. Asp. 178, 213 - 229. 